TRANSACTIONS OF THE

AMERICAN MATHEMATICAL SOCIETY

Volume 357, Number 1, Pages 129-150

S 0002-9947(04)03394-X

Article electronically published on January 23, 2004

\title{
A LYNDON-HOCHSCHILD-SERRE SPECTRAL SEQUENCE FOR CERTAIN HOMOTOPY FIXED POINT SPECTRA
}

\author{
ETHAN S. DEVINATZ
}

\begin{abstract}
Let $H$ and $K$ be closed subgroups of the extended Morava stabilizer group $G_{n}$ and suppose that $H$ is normal in $K$. We construct a strongly convergent spectral sequence

$$
H_{c}^{*}\left(K / H,\left(E_{n}^{h H}\right)^{*} X\right) \Rightarrow\left(E_{n}^{h K}\right)^{*} X,
$$

where $E_{n}^{h H}$ and $E_{n}^{h K}$ are the continuous homotopy fixed point spectra of Devinatz and Hopkins. This spectral sequence turns out to be an Adams spectral sequence in the category of $K(n)_{*}$-local $E_{n}^{h K}$-modules.
\end{abstract}

\section{INTRODUCTION}

Suppose that $Z$ is a spectrum and that $G$ is a group acting on $Z$ in some good point-set category of spectra. If $G$ is, say, profinite, one might hope to construct, for each closed subgroup $H$ of $G$, a "continuous homotopy fixed point spectrum", denoted (abusively) by $Z^{h H}$. There should be a "continuous homotopy fixed point spectral sequence"

$$
H_{c}^{*}\left(H, Z^{*} X\right) \Rightarrow\left[X, Z^{h H}\right]^{*}
$$

for all CW-spectra $X$, where $H_{c}^{*}(H$, ?) denotes the continuous cohomology of the profinite group $H$. This of course requires that $Z^{*} X$ has an appropriate topology on which $G$ acts continuously (see [19] or 6 Remark 1.7]). In addition, $Z^{h H}$ should have the expected functorial properties and should agree with the ordinary homotopy fixed point spectrum when $H$ is discrete.

We are interested in one very important example where such a construction is possible - namely, the action of the Morava stabilizer group $G_{n}$ on the Landweber exact spectrum $E_{n}$. First recall the definitions. For a fixed prime number $p$, the coefficient ring $E_{n^{*}}$ is $W \mathbb{F}_{p^{n}}\left[\left[u_{1}, \ldots, u_{n-1}\right]\right]\left[u, u^{-1}\right]$, where $\left|u_{i}\right|=0,|u|=-2$, and as usual $W \mathbb{F}_{p^{n}}$ denotes the ring of Witt vectors with coefficients in the field $\mathbb{F}_{p^{n}}$ of $p^{n}$ elements. $G_{n}=S_{n} \rtimes \mathrm{Gal}$, where $S_{n}$ denotes the automorphism group of the height $n$ Honda formal group law over $\mathbb{F}_{p^{n}}$, and Gal $\equiv \operatorname{Gal}\left(\mathbb{F}_{p^{n}} / \mathbb{F}_{p}\right)$ denotes the Galois group of the field extension $\mathbb{F}_{p^{n}} / \mathbb{F}_{p}$. Morava's theory implies that $G_{n}$ acts on $E_{n}$ by ring spectrum maps in the stable category, and technology developed by

Received by the editors September 13, 2002 and, in revised form, May 21, 2003.

2000 Mathematics Subject Classification. Primary 55N20; Secondary 55P43, 55T15.

Key words and phrases. Adams spectral sequence, continuous homotopy fixed point spectra, Morava stabilizer group.

The author was partially supported by a grant from the National Science Foundation.

(C)2004 American Mathematical Society 
Goerss, Hopkins, and Miller (see [9, [10, 11], [12, [16]) then implies that $E_{n}$ has a model on which $G_{n}$ acts before passage to the stable category, so that homotopy fixed point spectra may be formed. Moreover, $G_{n}$ is a profinite group - even a $p$-adic analytic group - and we constructed in [6] good continuous homotopy fixed point spectra for the action of this group on $E_{n}$. These continuous homotopy fixed point spectra are the homotopically significant spectra in this situation, since, for example, the continuous homotopy fixed point spectral sequences are generalized Adams spectral sequences and $E_{n}^{h G_{n}}=L_{K(n)} S^{0}$, the $K(n)_{*}$-localization of $S^{0}$.

Returning to the general situation, naturality of the continuous homotopy fixed point spectrum $Z^{h H}$ implies that it is acted upon by the group $N(H) / H$. If $F=$ $K / H$ is a finite subgroup of $N(H) / H$, we can form the ordinary homotopy $F$ fixed point spectrum $\left(Z^{h H}\right)^{h F}$, and we would expect this spectrum to be equivalent to the continuous homotopy fixed point spectrum $Z^{h K}$. This was proved for the action of $G_{n}$ on $E_{n}$ in [6]. If $F$ is closed but not finite, the situation becomes more problematic. Here the relevant spectrum is the continuous homotopy $F$ fixed points of $E_{n}^{h H}$. However, we do not have an intrinsic construction of such a spectrum-our construction of continuous homotopy fixed point spectra was specific to the action of $G_{n}$ on $E_{n}$. Yet it is clear that $\left(E_{n}^{h H}\right)^{h F}$ ought to be just $E_{n}^{h K}$. We can then ask whether there is a convergent spectral sequence

$$
H_{c}^{*}\left(K / H,\left(E_{n}^{h H}\right)^{*} X\right) \Rightarrow\left[X, E_{n}^{h K}\right]^{*} ;
$$

such a spectral sequence provides a way of using information about $E_{n}^{h H}$ to gain information about $E_{n}^{h K}$.

In this paper, we construct the spectral sequence (0.1) and prove that it is always strongly convergent. Our construction makes use of the highly structured results of [6]: if $G$ is a closed subgroup of $G_{n}, E_{n}^{h G}$ is a commutative $S^{0}$-algebra in the sense of $[8$ and the maps between these continuous homotopy fixed point spectra arising from functoriality are maps of commutative $S^{0}$-algebras. In particular, the inclusion $H \rightarrow K$ makes $E_{n}^{h H}$ a commutative $E_{n}^{h K}$-algebra. We can then form a $K(n)_{*}$-local $E_{n}^{h H}$-Adams resolution of $E_{n}^{h K}$ in the stable category of $E_{n}^{h K}$-modules, again in the sense of [8]. By neglect of structure, this Adams resolution gives us a diagram of cofibrations in the stable category. Map the CW-spectrum $X$ into this diagram to obtain the desired spectral sequence.

This paper is organized as follows. In $\S 1$, we recall the definition and properties of the stable category of $R$-modules, for $R$ a commutative $S^{0}$-algebra, and deal with some subtleties which will arise later. In $\S 2$, we give a general discussion of Adams spectral sequences in the stable category of $R$-modules. This material is a straightforward adaptation of work of Miller 15] and Bousfield 33; these Adams spectral sequences have also been considered by Baker and Lazerev [1. In $\S 3$, we identify the $E_{2}$-term of the aforementioned Adams spectral sequence with the continuous cohomology of $K / H$. Actually, we prove a more general result valid for $E_{n}^{h K}$-modules $X$; this is stated as Theorem 3.1 Along the way, we identify the algebra

$$
\pi_{*} L_{K(n)}\left(E_{n}^{h H} \wedge_{E_{n}^{h K}} E_{n}^{h H}\right)
$$

of homology cooperations. The strong convergence of this spectral sequence is established in $\S 4$, and finally, in the Appendix, we prove that, if $K / H$ is finite, the spectral sequence we have constructed agrees with the ordinary homotopy fixed point spectral sequence. 


\section{Categories of module spectra}

We use the framework of [8] in this paper. Our basic category of spectra is thus the category of $S^{0}$-modules $\mathcal{M}_{S^{0}}$; this category becomes the usual stable category, denoted $\mathcal{D}_{S^{0}}$, upon taking its homotopy category and inverting the weak equivalences. The advantage of the category of $S^{0}$-modules is that it is symmetric monoidal; that is, there is an associative, commutative, and unital smash product, denoted $\wedge$, and so one has the notion of $S^{0}$-algebras or commutative $S^{0}$-algebras. (These correspond to the earlier notions of $A_{\infty}$ and $E_{\infty}$ spectra, respectively.) If $R$ is an $S^{0}$-algebra, there is then the evident notion of a (left) $R$-module, and one can form the category $\mathcal{M}_{R}$ of such $R$-modules and $R$-module maps between them. If $R$ is commutative, a left $R$-module is the same as a right $R$-module, and one can define the smash product $M \wedge_{R} N$ of two $R$-modules to be the $R$-module given by the coequalizer diagram

$$
M \wedge R \wedge N \frac{\mu_{M} \wedge N}{\overrightarrow{M \wedge \mu_{N}}} M \wedge N \longrightarrow M \wedge_{R} N,
$$

where $\mu_{M}$ and $\mu_{N}$ are the module structure maps for $M$ and $N$. This smash product makes $\mathcal{M}_{R}$ a symmetric monoidal category with $R$ as the unit. The stable category $\mathcal{D}_{R}$ of $R$-modules, called the derived category in [8], is formed from the homotopy category of $\mathcal{M}_{R}$ by inverting the weak equivalences; i.e., those maps of $R$-modules, which, regarded as maps of spectra, induce isomorphisms of homotopy groups. Equivalently, $\mathcal{D}_{R}$ is the homotopy category of cell $R$-modules, again as defined in 8. The categories $\mathcal{M}_{R}$ and $\mathcal{D}_{R}$ have the same formal properties as the categories $\mathcal{M}_{S^{0}}$ and $\mathcal{D}_{S^{0}}$, and we shall use these for the most part without comment. However, the (derived) smash product in $\mathcal{D}_{R}$ will be the source of some complications later, and thus we give a more detailed discussion now.

If $M$ and $N$ are in $\mathcal{M}_{R}$, the derived smash product $M \Lambda_{R} N$ in $\mathcal{D}_{R}$ is defined to be $\Gamma M \wedge_{R} \Gamma N$, where $\Gamma M$ and $\Gamma N$ are cell $R$-modules weakly equivalent to $M$ and $N$ in $\mathcal{M}_{R}$. Of course, the canonical map $M \Lambda_{R} N \rightarrow M \wedge_{R} N$ need not be an equivalence in $\mathcal{D}_{R}$. If $f: R \rightarrow R^{\prime}$ is a map of commutative $S^{0}$-algebras, then there is an evident functor $f^{*}: \mathcal{M}_{R^{\prime}} \rightarrow \mathcal{M}_{R}$. Since $f^{*}$ preserves weak equivalences, we also have $f^{*}: \mathcal{D}_{R^{\prime}} \rightarrow \mathcal{D}_{R}$ and a natural transformation $f^{*} M \underline{\Lambda}_{R} f^{*} N \rightarrow f^{*}\left(M \Lambda_{R^{\prime}} N\right)$ in $\mathcal{D}_{R}$ for $M$ and $N$ in $\mathcal{M}_{R^{\prime}}$.

There are, however, certain situations where the map $M \triangle_{R} N \rightarrow M \wedge_{R} N$ is an equivalence. These will prove quite convenient in what follows.

Proposition 1.1 ([8, III, Theorem 3.8]). If $M$ is a cell $R$-module and $\phi: N \rightarrow N^{\prime}$ is a weak equivalence in $\mathcal{M}_{R}$, then $M \wedge_{R} \phi: M \wedge_{R} N \rightarrow M \wedge_{R} N^{\prime}$ is a weak equivalence. In particular, $M \wedge_{R} N \simeq M \Lambda_{R} N$ whenever $M$ is a cell $R$-module.

To state the next condition, we need a little more preparation. Recall that the category of commutative $R$-algebras has the structure of a model category (see $[8$. $\mathrm{VII}]$ ); we define a $q$-cofibrant commutative $R$-algebra to be a cofibrant object in this category. There is also the notion of a cell commutative $R$-algebra ([8] VII, Definition 4.11]), and any such object is $q$-cofibrant. These cell objects, however, do not in general have the homotopy type of cell $R$-modules.

Let $\overline{\mathcal{E}}_{R}$ denote the class of $R$-modules defined in [8, VII, 6]. Included in $\overline{\mathcal{E}}_{R}$ are all $R$-modules having the homotopy type of cell $R$-modules. The main reasons for interest in this class are the following results. 
Theorem 1.2 ([8 VII, Theorem 6.7]). Suppose that $R$ is a q-cofibrant commutative $S^{0}$-algebra and that $M_{i} \in \overline{\mathcal{E}}_{R}$ for $i=1, \ldots, k$. Then

$$
M_{1} \wedge_{R} \cdots \underline{\Lambda}_{R} M_{k} \rightarrow M_{1} \wedge_{R} \cdots \wedge_{R} M_{k}
$$

is an equivalence in $\mathcal{D}_{R}$.

Theorem 1.3 (8 VII, Theorem 6.5]). Suppose that $A$ is a q-cofibrant commutative $R$-algebra. Then $A$ is in $\overline{\mathcal{E}}_{R}$.

Since $\overline{\mathcal{E}}_{R}$ is closed under homotopy equivalences, finite $\wedge_{R}$-products, pushouts along cofibrations, and colimits of (countable) sequences of cofibrations, the next result is an easy consequence of Theorem 1.3

Proposition 1.4. Let $R^{\prime}$ be a q-cofibrant commutative $R$-algebra, and suppose the $R$-module $M$ has the homotopy type of a cell $R^{\prime}$-module (regarded as $R$-modules). Then $M \in \overline{\mathcal{E}}_{R}$.

These results will all be relevant to our work, since, in [6], we constructed the continuous homotopy fixed point spectra $E_{n}^{h G}$ to be cell commutative $S^{0}$-algebras.

We will also need some results from [8] on the Bousfield localization of $R$-modules. Suppose then that $F$ is a cell $R$-module. A map $f: X \rightarrow Y$ of $R$-modules is an $F_{*}^{R}$-equivalence if $F \wedge f: F \wedge_{R} X \rightarrow F \wedge_{R} Y$ is a weak equivalence, and an $R$-module $W$ is $F_{*}^{R}$-acyclic if $F \wedge_{R} W$ is weakly contractible. (We assume $F$ is a cell $R$-module so that we can work with the ordinary, as opposed to the derived, smash product.) An $R$-module $Y$ is $F_{*}^{R}$-local if $[W, Y]_{*}^{R}$ is trivial whenever $W$ is $F_{*}^{R}$-acyclic. Here $[W, Y]_{i}^{R}$ denotes the group of maps $\Sigma^{i} W \rightarrow Y$ in $\mathcal{D}_{R}$; we will also denote this group by $[W, Y]_{R}^{-i}$. Finally, $f: X \rightarrow Y$ is called the $F_{*}^{R}$-localization of $X$ if $f$ is an $F_{*}^{R}$-equivalence and $Y$ is $F_{*}^{R}$-local. If such a localization exists, it is unique up to canonical isomorphism in $\mathcal{D}_{R}$. It is proved in [8, VIII, 1] that $X$ always has an $F_{*}^{R}$-localization, which we denote $\lambda: X \rightarrow L_{F}^{R} X$. Moreover, if $R$ is a $q$-cofibrant $S^{0}$-algebra, e.g., $R=S^{0}$, and $X$ is a cell commutative $R$-algebra, then $\lambda$ can be constructed to be a map of cell commutative $R$-algebras and even to be natural in this category; i.e., the full subcategory of the category of commutative $R$-algebras and $R$-algebra maps whose objects are the cell commutative $R$-algebras.

If $K$ is a cell $S^{0}$-module, and $F=R \wedge K$ as $R$-modules, then a map $f: X \rightarrow$ $Y$ of $R$-modules is an $F_{*}^{R}$-equivalence if and only if it is a $K_{*}$-equivalence when regarded as a map of $S^{0}$-modules. Hence there is a canonical map $L_{F}^{R} X \rightarrow L_{K} X$ in $\mathcal{D}_{S^{0}}$, where we write $L_{K} X$ for $L_{K}^{S^{0}} X$. Furthermore, an $S^{0}$-module $W$ is $K_{*}$ acyclic if and only if $R \wedge W$ is $F_{*}^{R}$-acyclic; this implies that $L_{F}^{R} X$ is $K_{*}$-local and therefore that $L_{F}^{R} X \rightarrow L_{K} X$ is a weak equivalence. In particular, $K_{*}$-localization defines a functor and natural transformation on $\mathcal{D}_{R}$, and the $K_{*}$-localization of a commutative $R$-algebra can be taken to be a commutative $R$-algebra (for $R$ a $q$-cofibrant commutative $S^{0}$-algebra). The case with $K=K(n)$ and $R=E_{n}^{h G}$, for $G$ a closed subgroup of $G_{n}$, will be the case of interest in this paper.

\section{AdAms spectral SEQUEnCES IN CATEgories of MOdUle SPECTRA}

In this section we set up and discuss the convergence of Adams spectral sequences in localized categories of $R$-modules. Since we will be exclusively working in (full subcategories of) $\mathcal{D}_{R}$, we will, in this section only, write $\wedge_{R}$-instead of using the notation $\triangle_{R}$ introduced in $\S 1$ - for the derived smash product. 
Say that an $R$-module $E$ is a commutative homotopy $R$-algebra if it is provided with maps $\eta: R \rightarrow E$ and $\mu: E \wedge_{R} E \rightarrow E$ (in $\mathcal{D}_{R}$ ) such that the expected diagrams commute. Let $F$ be an $R$-module. Following Miller [15], we construct the $F_{*}^{R}$-local $E$-Adams resolution of an $\left(F_{*}^{R}\right.$-local $) R$-module $Y$ as an injective resolution of $Y$ for an appropriate injective class.

Definition 2.1. With notation as above, an $R$-module is $F_{*}^{R}$-local E-injective if it is a retract of $L_{F}^{R}\left(Z \wedge_{R} E\right)$ for some $R$-module $Z$. Such an $R$-module will also be called $E$-injective in $L_{F}^{R} \mathcal{D}_{R}$, where $L_{F}^{R} \mathcal{D}_{R}$ is the category of $F_{*}^{R}$-local $R$-modules. A sequence of $F_{*}^{R}$-local $R$-modules $X^{\prime} \rightarrow X \rightarrow X^{\prime \prime}$ is E-exact in $L_{F}^{R} \mathcal{D}_{R}$ if

$$
\left[X^{\prime}, I\right]_{*}^{R} \longleftarrow[X, I]_{*}^{R} \longleftarrow\left[X^{\prime \prime}, I\right]_{*}^{R}
$$

is exact for every $F_{*}^{R}$-local $E$-injective $R$-module $I$.

One can easily check that this defines an injective class in the sense of 14 in the category $L_{F}^{R} \mathcal{D}_{R}$. Thus each object $Y$ in $L_{F}^{R} \mathcal{D}_{R}$ has a resolution by $F_{*}^{R}$-local $E$-injective $R$-modules, unique up to chain homotopy.

If

$$
* \longrightarrow Y \longrightarrow I^{0} \longrightarrow I^{1} \longrightarrow I^{2} \longrightarrow \cdots
$$

is such a resolution, then we may construct a diagram

$$
Y=Y^{0} \searrow_{j \searrow I_{I^{0}}}^{\longleftarrow_{k}} Y_{j I^{1}} \coprod_{k}^{i} Y^{2} \longleftarrow \cdots
$$

of exact triangles; in this diagram the map

$$
L_{F}^{R}(j \wedge E): L_{F}^{R}\left(Y^{i} \wedge_{R} E\right) \rightarrow L_{F}^{R}\left(I^{i} \wedge_{R} E\right)
$$

is a split monomorphism. Conversely, a diagram of exact triangles as in (2.2) with each $I^{j} F_{*}^{R}$-local $E$-injective and $L_{F}^{R}\left(j \wedge_{R} E\right)$ split monic yields an injective resolution (2.1). Such a diagram is called an $F_{*}^{R}$-local E-Adams resolution of $Y$ or an $E$-Adams resolution of $Y$ in $L_{F}^{R} \mathcal{D}_{R}$ and is functorial up to chain homotopy.

By mapping an $R$-module $X$ into an $F_{*}^{R}$-local $E$-Adams resolution of $Y$, we obtain a spectral sequence, called the $F_{*}^{R}$-local E-Adams spectral sequence, with

$$
E_{1}^{s, t}=\left[X, I^{s}\right]_{t-s}^{R} .
$$

(This indexing follows the convention that $i$ and $j$ are maps of degree 0 , and $k$ is a map of degree -1.) When $R$ and $F$ are understood, we will write this spectral sequence as $E_{r}^{s, t}(X, Y ; E)$. Naturally, we hope that this spectral sequence converges strongly to $Y$; the rest of this section is devoted to establishing a criterion which guarantees this.

Following Bousfield [3. Definition 3.7], we define the class of $F_{*}^{R}$-local E-nilpotent $R$-modules to be the smallest class $\mathcal{C}$ of objects in $L_{F}^{R} \mathcal{D}_{R}$ such that

i. $L_{F}^{R} E \in \mathcal{C}$.

ii. $L_{F}^{R}\left(N \wedge_{R} X\right) \in \mathcal{C}$ whenever $N \in \mathcal{C}$ and $X \in \mathcal{D}_{R}$.

iii. If $X \rightarrow Y \rightarrow Z \rightarrow \Sigma X$ is a cofiber sequence in $L_{F}^{R} \mathcal{D}_{R}$ and two of $X, Y, Z$ are in $\mathcal{C}$, then so is the third.

iv. $\mathcal{C}$ is closed under retracts in $L_{F}^{R} \mathcal{D}_{R}$. 
Next, say that $Z \in L_{F}^{R} \mathcal{D}_{R}$ is $L_{F}^{R}-E_{*}^{R}$-local if $[W, Z]_{*}^{R}$ is trivial whenever $L_{F}^{R}\left(E \wedge_{R} W\right) \simeq *$. Observe that this is the same as saying that $Z$ is $\left(F \wedge_{R} E\right)_{*}^{R}$-local. The following result is proved just as in [3. Lemma 3.8].

Proposition 2.2. If $Z$ is $F_{*}^{R}$-local E-nilpotent, then $Z$ is $L_{F}^{R}$ - $E_{*}^{R}$-local.

The notion of an $F_{*}^{R}$-local $E$-nilpotent resolution of an object $Y \in L_{F}^{R} \mathcal{D}_{R}$ may also be defined along the lines of [3], and its uniqueness in an appropriate "procategory" may also be established.

These resolutions are related to Adams spectral sequences as follows. Consider the Adams resolution (2.2), and let $Y_{s}$ be the cofiber of $i^{(s+1)}: Y^{s+1} \rightarrow Y$. We can then construct commutative diagrams

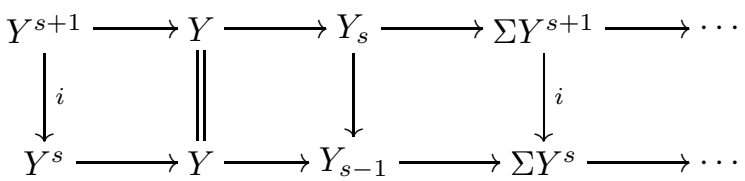

of cofibration sequences, as well as a diagram

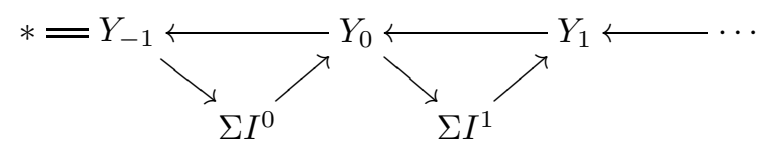

of exact triangles in $\mathcal{D}_{R}$. The spectral sequence obtained by mapping an $R$-module $X$ into this diagram yields the $F_{*}^{R}$-local $E$-Adams spectral sequence. Moreover, the tower $\left\{Y_{s}\right\}_{s \geq 0}$ under $Y$ is an $F_{*}^{R}$-local $E$-nilpotent resolution of $Y$ (see [3, Lemma 5.7]). Thus, if $Y$ is itself $F_{*}^{R}$-local $E$-nilpotent, then the constant tower $\{Y\}$ is proisomorphic to $\left\{Y_{s}\right\}$. This implies the following convergence result (see [3, Theorem $6.10])$.

Proposition 2.3. If $Y$ is $F_{*}^{R}$-local E-nilpotent, then the $F_{*}^{R}$-local E-Adams spectral sequence converges strongly and conditionally to $[X, Y]_{*}^{R}$, for any $R$-module $X$. In addition, there exists $s_{0}$ such that $E_{\infty}^{s, *}(X, Y ; E)=0$ for all $s>s_{0}$ and any $R$ module $X$.

Remark 2.4. We are using the notions of strong and conditional convergence as given in Boardman [2] applied to the unrolled exact couple

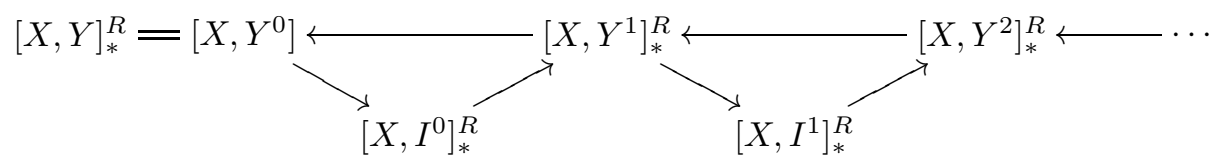

Note that in the presence of conditional (resp. strong) convergence, strong (resp.

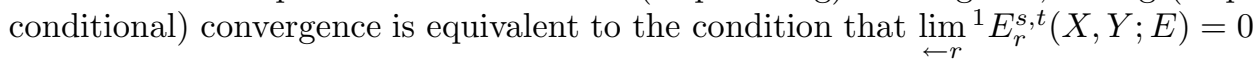
for all $s, t([2$, Theorem 7.3]).

The next result is a partial converse to Proposition 2.3 and will be useful to us later.

Proposition 2.5. Suppose that $Y$ is $L_{F}^{R}-E_{*}^{R}$-local and that there exist $s_{0}$ and $r_{0}$ such that for all $R$-modules $X$, the $F_{*}^{R}$-local $E$-Adams spectral sequence satisfies $E_{r_{0}}^{s, *}(X, Y ; E)=0$ whenever $s>s_{0}$. Then $Y$ is $F_{*}^{R}$-local E-nilpotent. 
Proof. Since $\lim _{\leftarrow r}^{1} E_{r}^{s, t}(X, Y ; E)=0$ for all $s, t$, it follows from [2, Theorem 7.4], applied to the unrolled exact couple

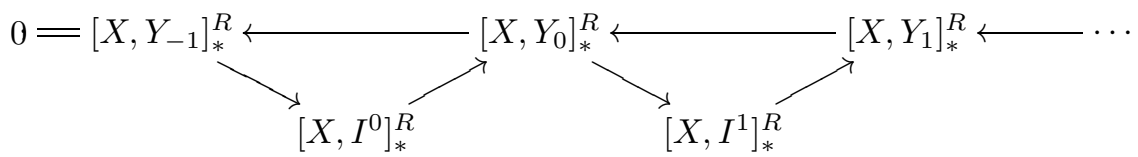

that

i. $\lim _{\leftarrow s}^{1}\left[X, Y_{s}\right]_{*}^{R}=0$ for all $R$-modules $X$,

ii. $F_{s, t}(X, Y ; E) / F_{s+1, t+1}(X, Y ; E) \stackrel{\approx}{\longrightarrow} E_{\infty}^{s, t}(X, Y ; E)$,

where

$$
\begin{aligned}
F_{s, t}(X, Y ; E) & =\operatorname{ker}\left(\lim _{\leftarrow i}\left[X, Y_{i}\right]_{t-s}^{R} \longrightarrow\left[X, Y_{s-1}\right]_{t-s}^{R}\right) \\
& =\operatorname{ker}\left(\left[X, \underset{\leftarrow i}{\operatorname{holim}} Y_{i}\right]_{t-s}^{R} \longrightarrow\left[X, Y_{s-1}\right]_{t-s}^{R}\right) .
\end{aligned}
$$

The horizontal vanishing line now implies that $F_{s_{0}+1, *}(X, Y ; E)=0$. Since $X$ is arbitrary, this in turn implies that the map $p: \underset{\leftarrow i}{\operatorname{holim}} Y_{i} \rightarrow Y_{s_{0}}$ is the inclusion of a summand in $\mathcal{D}_{R}$; in particular, $\underset{\leftarrow i}{\operatorname{holim}} Y_{i}$ is $F_{*}^{R}$-local $E$-nilpotent.

Now consider the map $\iota: Y \rightarrow \stackrel{\leftarrow}{h}$ holim $Y_{s}$ which lifts the canonical maps $Y \rightarrow Y_{s}$. (This map is unique since $\lim _{\leftarrow}^{1}\left[Y, Y_{s}\right]_{*}^{R}=0$.) $\iota$ is a map between $L_{F}^{R} E_{*}^{R}$ local objects; it is therefore an equivalence if and only if

$$
L_{F}^{R}\left(E \wedge_{R} \iota\right): L_{F}^{R}\left(E \wedge_{R} Y\right) \rightarrow L_{F}^{R}\left(E \wedge_{R} \underset{\leftarrow s}{\operatorname{holim}} Y_{s}\right)
$$

is an equivalence. We will prove that $L_{F}^{R}\left(E \wedge_{R} \iota\right)$ is an equivalence, and hence $Y$ is $F_{*}^{R}$-local E-nilpotent.

Begin by observing that $L_{F}^{R}\left(E \wedge_{R} Y\right)$ is $F_{*}$-local $E$-injective, so that

$$
E_{\infty}^{s, t}\left(X, L_{F}^{R}\left(E \wedge_{R} Y\right) ; E\right)=E_{2}^{s, t}=\left\{\begin{array}{cc}
{\left[X, L_{F}^{R}\left(E \wedge_{R} Y\right)\right]_{t}^{R}} & s=0, \\
0 & s \neq 0 .
\end{array}\right.
$$

Since $L_{F}^{R}\left(E \wedge_{R}\right.$ ?) applied to an $F_{*}^{R}$-local $E$-Adams resolution of $Y$ is an $F_{*}^{R}$-local $E$ Adams resolution of $L_{F}^{R}\left(E \wedge_{R} Y\right)$, it follows as above that $\lim _{\leftarrow s}^{1}\left[X, L_{F}^{R}\left(E \wedge_{R} Y_{s}\right)\right]_{*}^{R}=0$ for all $X$. Hence there is a canonical map

$$
L_{F}^{R}\left(E \wedge_{R} Y\right) \rightarrow \underset{\leftarrow s}{\operatorname{holim}} L_{F}^{R}\left(E \wedge_{R} Y_{s}\right)
$$

and this map is an equivalence.

Now there is also a canonical map

$$
j: L_{F}^{R}\left(E \wedge_{R} \underset{\leftarrow s}{\operatorname{holim}} Y_{s}\right) \rightarrow \underset{\leftarrow s}{\operatorname{holim}} L_{F}^{R}\left(E \wedge_{R} Y_{s}\right),
$$

whence the composition

$$
L_{F}\left(E \wedge_{R} Y\right) \stackrel{L_{F}^{R}(E \wedge \iota)}{\longrightarrow} L_{F}^{R}\left(E \wedge_{R} \underset{\leftarrow s}{\operatorname{holim}} Y_{s}\right) \stackrel{j}{\longrightarrow} \underset{\leftarrow s}{\operatorname{holim}} L_{F}^{R}\left(E \wedge_{R} Y_{s}\right)
$$

is an equivalence. Thus $\pi_{*} j$ is an epimorphism.

On the other hand, choose $r_{0}: Y_{s_{0}} \rightarrow \underset{\leftarrow s}{\operatorname{holim}} Y_{s}$ so that $r_{0} \circ p=i d$, and let $r$ be the composition

$$
\underset{\leftarrow s}{\operatorname{holim}} L_{F}^{R}\left(E \wedge_{R} Y_{s}\right) \rightarrow L_{F}^{R}\left(E \wedge_{R} Y_{s_{0}}\right) \stackrel{L_{F}^{R}\left(E \wedge r_{0}\right)}{\longrightarrow} L_{F}^{R}\left(E \wedge_{R} \underset{\leftarrow s}{\operatorname{holim}} Y_{s}\right) .
$$


Then $r \circ j$ is the identity, so $\pi_{*} j$ is a monomorphism and therefore an isomorphism. This implies that $L_{F}^{R}\left(E \wedge_{R} \iota\right)$ is an equivalence, completing the proof.

\section{IDENTIFICATION OF THE $E_{2}$-TERM}

For the rest of this paper, the integer $n \geq 1$ will be fixed, so we will delete the subscript $n$ from $E_{n}$ or any of its continuous homotopy fixed point spectra. We will also write $L_{K(n)}$ as $\widehat{L}$, but will retain the notation $K(n)$, reserving the notation $K$ for closed subgroups of $G_{n}$.

Let $H$ and $K$ be closed subgroups of $G_{n}$ with $H$ normal in $K$. The main result of [6] constructs $K(n)_{*}$-local cell commutative $S^{0}$-algebras $E^{h H}$ and $E^{h K}$ which are to be interpreted as continuous homotopy fixed point spectra. The inclusion of $H$ in $K$ induces a map $E^{h K} \rightarrow E^{h H}$ of commutative $S^{0}$-algebras, so that $E^{h H}$ is a commutative $E^{h K}$-algebra. Moreover, there is an action of $K / H$ on $E^{h H}$ by $E^{h K}$-algebra maps, whose construction we now recall.

If

$$
G_{n}=U_{0} \supsetneq U_{1} \supsetneq U_{2} \supsetneq \cdots \supsetneq U_{i} \supsetneq \cdots
$$

is a sequence of open normal subgroups of $G_{n}$ with $\bigcap_{i} U_{i}=\{e\}$, then

$$
E^{h G}=\widehat{L}\left(\underset{\rightarrow i}{\operatorname{holim}} E^{h\left(U_{i} G\right)}\right)
$$

for any closed subgroup $G$ of $G_{n}$. Each left $G_{n}$-map $\rho: G_{n} / H U_{i} \rightarrow G_{n} / H U_{i}$ making the diagram

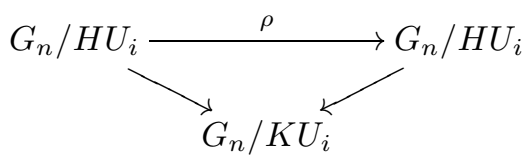

commute induces an $E^{h\left(K U_{i}\right)}$-algebra map $\mathbf{F}(\rho): E^{h\left(U_{i} H\right)} \rightarrow E^{h\left(U_{i} H\right)} \underline{6}$, Theorem 1]. If $g H$ is an element of $K / H$, define such an automorphism of $G_{n} / H U_{i}$ by sending $x H U_{i}$ to $x g H U_{i}$. Then pass to homotopy colimits to obtain an $E^{h K_{-}}$ algebra automorphism of $E^{h H}$. Under the isomorphism

$$
\pi_{*} \widehat{L}\left(E^{h H} \wedge E\right) \stackrel{\approx}{\longrightarrow} \operatorname{Map}_{c}\left(H \backslash G_{n}, E_{*}\right)
$$

of $\pi_{*} \widehat{L}(E \wedge E) \approx \operatorname{Map}_{c}\left(G_{n}, E_{*}\right)$-comodules ([․, Theorem 2])-here $H \backslash G_{n}$ denotes the space of right cosets $H x$ in $G_{n}$-this action of $g H \equiv \bar{g}$ induces the map

$$
\bar{g}_{*}: \operatorname{Map}_{c}\left(H \backslash G_{n}, E_{*}\right) \rightarrow \operatorname{Map}_{c}\left(H \backslash G_{n}, E_{*}\right)
$$

given by

$$
\bar{g}_{*}(f)(H x)=f\left(H g^{-1} x\right) .
$$

We can now state the main results of this paper. To make the notation less cumbersome, we will write $E(G)$ for $E^{h G}$ when this object appears as a sub or superscript.

Theorem 3.1. Let $X$ be an $E^{h K}$-module. The $K(n)_{*}$-local $E^{h H}$-Adams spectral sequence $E_{r}^{s, t}\left(X, E^{h K} ; E^{h H}\right)$ in $\mathcal{D}_{E(K)}$ has $E_{2}$-term naturally isomorphic to $H_{c}^{s}\left(K / H,\left[X, E^{h H}\right]_{t}^{E(K)}\right)$. 
Remark 3.2. By $K(n)_{*}$-local, we really mean $\left(K(n) \wedge E^{h K}\right)_{*}^{E(K)}$-local, but, by the discussion in $\S 1$, the functors $L_{K(n)}$ and $L_{K(n) \wedge E(K)}^{E(K)}$ are naturally equivalent when restricted to $E^{h K}$-modules.

By Proposition 2.3, the next result implies that this spectral sequence converges strongly to $\left[X, E_{n}^{h H}\right]_{*}^{E(K)}$.

Theorem 3.3. Every $E^{h K}$-module is $K(n)_{*}$-local $E^{h H}$-nilpotent in $\mathcal{D}_{E(K)}$.

Corollary 3.4. There is a strongly convergent spectral sequence

$$
H_{c}^{s}\left(K / H,\left(E^{h H}\right)^{*} Z\right) \Rightarrow\left(E^{h K}\right)^{t+s}(Z),
$$

valid for any object $Z$ in the stable category.

Proof. If $Z$ is a cell $S^{0}$-module, apply Theorem 3.1 to $X=E^{h K} \wedge Z$ and use the fact that

$$
[X, M]^{E(K)}=[Z, M]
$$

whenever $M$ is an $E^{h K}$-module.

To prove Theorem 3.1 we must first make sense of $H_{c}^{s}\left(K / H,\left[X, E^{h H}\right]_{t}^{E(K)}\right)$. Since $K / H$ is a $p$-analytic profinite group [7, Theorem 9.6], $H_{c}^{s}(K / H, M)$ makes good sense whenever $M$ is a profinite continuous $\mathbb{Z}_{p}[[K / H]]$-module: it can be defined as $\lim _{\leftarrow \alpha} H_{c}^{s}\left(K / H, M_{\alpha}\right)$, where $M=\lim _{\leftarrow \alpha} M_{\alpha}$ with each $M_{\alpha}$ a finite discrete $K / H$-module (see [6, Remark 1.3]). The action of $K / H$ on $E^{h H}$ induces an action on $\left[X, E^{h H}\right]_{t}^{E(K)}$; we thus need to provide $\left[X, E^{h H}\right]_{t}^{E(K)}$ with a natural topology so that it becomes a profinite continuous $\mathbb{Z}_{p}[[K / H]]$-module.

If $I$ is the multi-index $\left(i_{0}, \ldots, i_{n-1}\right)$, let $M(I)$ denote a finite spectrum with

$$
B P_{*} M(I)=B P_{*} /\left(p^{i_{0}}, v_{1}^{i_{1}}, \ldots, v_{n-1}^{i_{n-1}}\right),
$$

provided such a spectrum exists.

Lemma 3.5. Let $X$ be a finite cell $E^{h K}$-module. Then $\left[X, E^{h H} \wedge M(I)\right]_{*}^{E(K)}$ is a finite discrete $K / H$-module in each degree.

Proof. It suffices to show that $\left[X, E^{h H} \wedge M(I)\right]_{*}^{E(K)}$ is a finite discrete $K / H$-module in each degree when $X$ is a "sphere $E^{h K}$-module" ([8, III,2]). Hence we need only show that $\pi_{*}\left(E^{h H} \wedge M(I)\right)$ is a finite discrete $K / H$-module in each degree.

Consider the continuous homotopy fixed point spectral sequence

$$
E_{2}^{* * *}=H_{c}^{*}\left(H, E_{*} /\left(p^{i_{0}}, \ldots, v_{n-1}^{i_{n-1}}\right)\right) \Rightarrow \pi_{*}\left(E^{h H} \wedge M(I)\right)
$$

from [6, Theorem 2]. Since $E_{*} /\left(p^{i_{0}}, \ldots, v_{n-1}^{i_{n-1}}\right)$ is finite in each degree, it follows that $H_{c}^{*}\left(H, E_{*} /\left(p^{i_{0}}, \ldots, v_{n-1}^{i_{n-1}}\right)\right)$ is finite in each bidegree (see e.g. [6] proof of Lemma 4.21]. But $E_{\infty}^{* * *}$ has a horizontal vanishing line since $E^{h H}$ is $K(n)_{*}$-local $E$ nilpotent (in the ordinary stable category) [6, Proposition A.3], and this spectral sequence is the $K(n)_{*}$-local $E$-Adams spectral sequence. This allows us to conclude that $\pi_{*}\left(E^{h H} \wedge M(I)\right)$ is finite in each degree.

Furthermore,

$$
\pi_{*}\left(E^{h H} \wedge M(I)\right)=\lim _{\rightarrow i} \pi_{*}\left(E^{h H U_{i}} \wedge M(I)\right),
$$


where the $U_{i}$ 's are as in (3.1). But $\pi_{*}\left(E^{h H U_{i}} \wedge M(I)\right)$ is a discrete $K / H$ module, since $K \cap U_{i}$ acts trivially on $E^{h H U_{i}}$. Hence $\pi_{*}\left(E^{h H} \wedge M(I)\right)$ is a discrete $K / H$ module, and the proof is complete.

Proposition 3.6. If $X$ is any cell $E^{h K}$-module, then

$$
\left[X, E^{h H}\right]_{*}^{E(K)}=\lim _{\leftarrow \alpha, I}\left[X_{\alpha}, E^{h H} \wedge M(I)\right]_{*}^{E(K)}
$$

where the $X_{\alpha}$ range over the finite $E^{h K}$-cell subcomplexes of $X$, and the $M(I)$ range over a sequence of multi-indices such that $\widehat{L} S^{0}=\underset{\leftarrow I}{\operatorname{holim}} M(I)$. With the topology of this inverse limit of discrete spaces, $\left[X, E^{h H}\right]_{t}^{E(K)}$ is therefore a profinite continuous $K / H$-module.

Proof. Since $E^{h H}$ is $K(n)_{*}$-local,

$$
E^{h H} \stackrel{\approx}{\longrightarrow} \widehat{L} E^{h H}=\underset{\leftarrow I}{\operatorname{holim}} E^{h H} \wedge M(I) .
$$

It therefore follows from the previous lemma that

$$
\left[X_{\alpha}, E^{h H}\right]_{*}^{E(K)}=\lim _{\leftarrow I}\left[X_{\alpha}, E^{h H} \wedge M(I)\right]_{*}^{E(K)}
$$

and in particular that $\left[X_{\alpha}, E^{h H}\right]_{*}^{E(K)}$ is a profinite group in each degree. Hence

$$
\begin{aligned}
{\left[X, E^{h H}\right]_{*}^{E(K)} } & =\lim _{\leftarrow \alpha}\left[X_{\alpha}, E^{h H}\right]_{*}^{E(K)} \\
& =\lim _{\leftarrow \alpha, I}\left[X_{\alpha}, E^{h H} \wedge M(I)\right]_{*}^{E(K)},
\end{aligned}
$$

completing the proof.

Now consider the $E^{h H}$-injective resolution $* \rightarrow E^{h K} \stackrel{\eta}{\rightarrow} I^{0} \stackrel{d}{\rightarrow} I^{1} \stackrel{d}{\rightarrow} \cdots$ in $\widehat{L} \mathcal{D}_{E(K)}$ given by

$$
I^{j}=\widehat{L}(\underbrace{E^{h H} \triangle_{E(K)} \cdots \Lambda_{E(K)} E^{h H}}_{j+1 \text { copies }})
$$

with $\eta: E^{h K} \rightarrow E^{h H}$ the inclusion and $d: I^{j} \rightarrow I^{j+1}$ given by $d=\sum_{i=0}^{j+1}(-1)^{i} d^{i}$, where

$$
d^{i}=\widehat{L}\left(\left(E^{h H}\right)^{(i)} \Lambda_{E(K)} \eta \underline{\Lambda}_{E(K)}\left(E^{h H}\right)^{(j+1-i)}\right) .
$$

(Recall from $\S 1$ that $\Lambda_{E(K)}$ denotes the smash product in $\mathcal{D}_{E(K)}$.) Since iterated smash products will be ubiquitous, let us write

$$
\Gamma^{j}(A ; R) \equiv \underbrace{A \triangle_{R} A \underline{\Lambda}_{R} \cdots \triangle_{R} A}_{j+1 \text { copies }}
$$

whenever $A$ is a commutative $R$-algebra (and $R$ is a commutative $S^{0}$-algebra). Thus $\widehat{L} \Gamma^{j}\left(E^{h H} ; E^{h K}\right)=I^{j}$.

Theorem 3.1 will follow easily from the next result.

Theorem 3.7. Let $X$ be a cell $E^{h K}$-module, and let

$$
T_{j}:\left[X, I^{j}\right]_{*}^{E(K)} \rightarrow \operatorname{Map}_{c}(\underbrace{K / H \times \cdots \times K / H}_{j \text { copies }},\left[X, E^{h H}\right]_{*}^{E(K)})
$$


be defined by taking $T_{j}(f)\left(\bar{g}_{1}, \ldots, \bar{g}_{j}\right)$ to be the composition

$$
X \stackrel{f}{\rightarrow} I^{j} \stackrel{\bar{g}_{1}^{-1} \wedge \cdots \wedge \bar{g}_{j}^{-1} \wedge E^{h H}}{\longrightarrow} I^{j} \stackrel{\mu}{\rightarrow} E^{h H},
$$

where we write $\bar{g}: E^{h H} \rightarrow E^{h H}$ for the action of $\bar{g} \in K / H$ on $E^{h H}$. Then $T_{j}$ is an isomorphism.

Remark 3.8. It is not obvious that $T_{j}(f)$ is even a continuous map from $(K / H)^{j}$ to $\left[X, E^{h H}\right]_{*}^{E(K)}$. This will be proven along with the theorem.

Assuming Theorem 3.7, we can now prove half of our main result.

Proof of Theorem 3.1. With $T_{j}$ as in 3.7, we have

$$
\begin{aligned}
T_{j+1}(d f)\left(\bar{g}_{1}, \ldots, \bar{g}_{j+1}\right)=\sum_{i=0}^{j}(-1)^{i} T_{j}(f)\left(\bar{g}_{1}, \ldots, \widehat{\bar{g}}_{i+1}, \ldots, \bar{g}_{j+1}\right) \\
+(-1)^{j+1} \bar{g}_{j+1}^{-1}\left[T_{j}(f)\left(\bar{g}_{1} \bar{g}_{j+1}^{-1}, \ldots, \bar{g}_{j} \bar{g}_{j+1}^{-1}\right)\right]
\end{aligned}
$$

for all $f \in I^{j}$.

Define, for each discrete $K / H$-module $M$, a cochain complex $C^{*}(K / H, M)$ by

$$
C^{j}(K / H, M)=\operatorname{Map}_{c}\left((K / H)^{j}, M\right)
$$

with differential $d: C^{j}(K / H, M) \rightarrow C^{j+1}(K / H, M)$ given by

$$
\begin{aligned}
(d f)\left(\bar{g}_{1}, \ldots, \bar{g}_{j+1}\right)= & \sum_{i=0}^{j}(-1)^{i} f\left(\bar{g}_{1}, \ldots, \widehat{\bar{g}}_{i+1}, \ldots, \bar{g}_{j+1}\right) \\
& +(-1)^{j+1} \bar{g}_{j+1}^{-1}\left[f\left(\bar{g}_{1} \bar{g}_{j+1}^{-1}, \ldots, \bar{g}_{j} \bar{g}_{j+1}^{-1}\right)\right] .
\end{aligned}
$$

Then $H^{*}\left(C^{*}(K / H, ?)\right)$ defines an effaceable $\delta$-functor, and, since $H^{0}\left(C^{*}(K / H, M)\right)$ $=M^{K / H}$, it follows that

$$
H^{*}\left(C^{*}(K / H, M)\right)=H_{c}^{*}(K / H, M) .
$$

Of course, $C^{*}(K / H, M)$ can also be defined if $M=\lim _{\leftarrow \alpha} M_{\alpha}$ is a profinite continuous $\mathbb{Z}_{p}[[K / H]]$-module, and the same proof as in [6, Lemma 4.21] applies to show that

$$
\begin{aligned}
H^{*}\left(C^{*}(K / H, M)\right) & =\lim _{\leftarrow \alpha} H^{*}\left(C^{*}\left(K / H, M_{\alpha}\right)\right) \\
& =\lim _{\leftarrow \alpha} H_{c}^{*}\left(K / H, M_{\alpha}\right) \\
& =H_{c}^{*}(K / H, M) .
\end{aligned}
$$

Since 3.4 identifies the cochain complex $\left[X, I^{*}\right]_{*}^{E(K)}$ with $C^{*}\left(K / H,\left[X, E^{h H}\right]_{*}^{E(K)}\right)$, it therefore follows that

$$
E_{2}^{s, t}\left(X, E^{h K} ; E^{h H}\right)=H_{c}^{s}\left(K / H,\left[X, E^{h H}\right]_{*}^{E(K)}\right),
$$

as desired.

Another consequence of Theorem 3.7 is the identification of the ring of homology cooperations (in the $K(n)_{*}$-local category) of $E^{h H}$ regarded as an $E^{h K}$-algebra.

Corollary 3.9. Let $K$ and $H$ be closed subgroups of $G_{n}$ with $H$ normal in $K$. Then

$$
\pi_{*} \widehat{L}\left(E^{h H} \triangle_{E(K)} E^{h H}\right)=\operatorname{Map}_{c}\left(K / H, E_{*}^{h H}\right) .
$$


The action $\bar{g}_{*}^{L}$ of $\bar{g} \in K / H$ on the left factor of $\pi_{*} \widehat{L}\left(E^{h H} \triangle_{E(K)} E^{h H}\right)$ is given by

$$
\bar{g}_{*}^{L}(f)(\bar{x})=f\left(\bar{g}^{-1} \bar{x}\right),
$$

and the action $\bar{g}_{*}^{R}$ of $\bar{g}$ on the right factor of $\pi_{*} \widehat{L}\left(E^{h H} \triangle_{E(K)} E^{h H}\right)$ is given by

$$
\bar{g}_{*}^{R}(f)(\bar{x})=\bar{g}_{*} f(\bar{x} \bar{g}),
$$

for any $f \in \operatorname{Map}_{c}\left(K / H, E_{*}^{h H}\right)$.

The rest of this section is devoted to proving Theorem 3.7 We begin by computing $\pi_{*} \widehat{L}\left(I^{j} \triangle E\right)$. (Note here that, in taking the derived smash product with $E$, $I^{j}$ is regarded as an object in $\mathcal{D}_{S^{0}}$.) This will require a number of lemmas; we first identify $\widehat{L}\left(I^{j} \wedge E\right)$ with $\widehat{L}\left[\Gamma^{j}\left(\widehat{L}\left(E^{h H} \wedge E\right) ; \widehat{L}\left(E^{h K} \wedge E\right)\right)\right]$.

Remark 3.10. The proof of Theorem 3.7 will be seen to reduce to the case where $H$ and $K$ are open in $G_{n}$, and thus we only need to understand $\widehat{L}\left(I^{j} \wedge E\right)$ in this case. However, we will carry out our analysis of $\widehat{L}\left(I^{j} \wedge E\right)$ in the general case, since some of our work along the way will be used in the next section.

Lemma 3.11. There is a canonical equivalence $I^{j} \wedge E \simeq \Gamma^{j}\left(E^{h H} \wedge E ; E^{h K} \wedge E\right)$ in $\mathcal{D}_{S^{0}}$.

Proof. This result would be essentially immediate if derived smash products were not involved; their presence, however, forces us to do a little more work.

Replace $E^{h H}$ by a weakly equivalent $q$-fibrant commutative $E^{h K}$-algebra $\bar{E}^{h H}$. Then, since $E^{h K}$ is a $q$-cofibrant commutative $S^{0}$-algebra, it follows by Theorems 1.2 and 1.3 that $I^{j}$ is represented in $\mathcal{D}_{E^{h K}}$ by (the ordinary smash product) $\bar{E}^{h H} \wedge_{E(K)} \cdots \wedge_{E(K)} \bar{E}^{h H} \cdot \bar{E}^{h H} \wedge_{E(K)} \cdots \wedge_{E(K)} \bar{E}^{h H}$ is the coproduct of $j+1$ copies of $\bar{E}^{h H}$ in the category of commutative $E^{h K}$-algebras and is therefore $q$-cofibrant as well. Again using the fact that $E^{h K}$ is a $q$-cofibrant commutative $S^{0}$-algebra, we conclude that $\bar{E}^{h H} \wedge_{E(K)} \cdots \wedge_{E(K)} \bar{E}^{h H}$ is also a $q$-cofibrant commutative $S^{0}$ algebra. Since $E$ is a $q$-cofibrant commutative $S^{0}$-algebra, $I^{j} \triangle E$ is thus represented by $\left(\bar{E}^{h H} \wedge_{E(K)} \cdots \wedge_{E(K)} \bar{E}^{h H}\right) \wedge E$ in $\mathcal{D}_{S^{0}}$. But

$$
\begin{aligned}
\left(\bar{E}^{h H} \wedge_{E(K)} \cdots \wedge_{E(K)} \bar{E}^{h H}\right) \wedge E & =\left(\bar{E}^{h H} \wedge_{E(K)} \cdots \wedge_{E(K)} \bar{E}^{h H}\right) \wedge\left(E \wedge_{E} \cdots \wedge_{E} E\right) \\
& =\left(\bar{E}^{h H} \wedge E\right) \wedge_{E(K) \wedge E} \cdots \wedge_{E(K) \wedge E}\left(\bar{E}^{h H} \wedge E\right),
\end{aligned}
$$

and $\bar{E}^{h H} \wedge E$ is a $q$-cofibrant commutative algebra over the $q$-cofibrant commutative $S^{0}$-algebra $E(K) \wedge E$. Therefore, $\left(\bar{E}^{h H} \wedge_{E(K)} \cdots \wedge_{E(K)} \bar{E}^{h H}\right) \wedge E$ represents $\Gamma^{j}\left(E^{h H} \wedge E ; E^{h K} \wedge E\right)$ in $\mathcal{D}_{S^{0}}$, completing the proof.

Lemma 3.12. The canonical map

$$
\Gamma^{j}\left(E^{h H} \wedge E ; E^{h K} \wedge E\right) \rightarrow \Gamma^{j}\left(\widehat{L}\left(E^{h H} \wedge E\right) ; \widehat{L}\left(E^{h K} \wedge E\right)\right)
$$

is a $K(n)_{*}$-equivalence in $\mathcal{D}_{S^{0}}$. Therefore,

$$
\widehat{L}\left(I^{j} \wedge E\right) \simeq \widehat{L}\left[\Gamma^{j}\left(\widehat{L}\left(E^{h H} \wedge E\right) ; \widehat{L}\left(E^{h K} \wedge E\right)\right)\right] .
$$

Proof. First recall from $\S 1$ that $E^{h H} \wedge E$ and $E^{h K} \wedge E$ are cell commutative $S^{0}$ algebras, so that $\widehat{L}\left(E^{h H} \wedge E\right)$ and $\widehat{L}\left(E^{h K} \wedge E\right)$ are cell commutative $S^{0}$-algebras, 
and $\widehat{L}\left(E^{h H} \wedge E\right)$ is a commutative $\widehat{L}\left(E^{h K} \wedge E\right)$-algebra. In addition, $\widehat{L}\left(E^{h H} \wedge E\right)$ is a commutative $\left(E^{h H} \wedge E\right)$-algebra, so we have the factorization

$$
\begin{aligned}
\Gamma^{j}\left(E^{h H} \wedge E ; E^{h K} \wedge E\right) & \stackrel{v}{\rightarrow} \Gamma^{j}\left(\widehat{L}\left(E^{h H} \wedge E\right) ; E^{h K} \wedge E\right) \\
& \stackrel{w}{\rightarrow} \Gamma^{j}\left(\widehat{L}\left(E^{h H} \wedge E\right) ; \widehat{L}\left(E^{h K} \wedge E\right)\right) .
\end{aligned}
$$

$K(n)$ is Bousfield equivalent to $K(n)^{(j+1)}$; we will therefore first prove that $v$ is a $K(n)_{*}$-equivalence by proving that it is a $K(n)_{*}^{(j+1)}$-equivalence. Now

$$
\Gamma^{j}\left(E^{h H} \wedge E ; E^{h K} \wedge E\right) \wedge K(n)^{(j+1)}=\Gamma^{j}\left(E^{h H} \wedge E \wedge K(n) ; E^{h K} \wedge E\right)
$$

with a similar statement for $\Gamma^{j}\left(\widehat{L}\left(E^{h H} \wedge E\right) ; E^{h K} \wedge E\right)$. (We are of course assuming that $K(n)$ is a cell $S^{0}$-module.) Since $E^{h H} \wedge E \wedge K(n) \stackrel{\simeq}{\rightarrow} \widehat{L}\left(E^{h H} \wedge E\right) \wedge K(n)$, it is now immediate that $v$ is a $K(n)_{*}^{(j+1)}$-equivalence.

That $w$ is a $K(n)_{*}$-equivalence follows by induction from the next result, completing the proof.

Lemma 3.13. Let $F$ be a cell $S^{0}$-module, let $R$ be a cell commutative $S^{0}$-algebra, and let $L R$ be the $F_{*}$-localization of $R$ (constructed to be an $R$-algebra and a cell commutative $S^{0}$-algebra.) If $M$ and $N$ are any LR-modules, then the canonical map $M \underline{\Lambda}_{R} N \rightarrow M \underline{\Lambda}_{L R} N$ is an $F_{*}$-equivalence.

Proof. We will prove that

$$
M \triangle_{R}(N \wedge F) \rightarrow M \triangle_{L R}(N \wedge F)
$$

is an equivalence; this is equivalent to the conclusion of the lemma.

Let $\Gamma M \rightarrow M$ (resp. $\Gamma N \rightarrow N)$ be a weak equivalence of $L R$-modules, where $\Gamma M(\operatorname{resp} . \Gamma N)$ is a cell $L R$-module, and let $\Gamma^{\prime} M \rightarrow \Gamma M\left(\operatorname{resp} . \Gamma^{\prime} N \rightarrow \Gamma N\right)$ be a weak equivalence of $R$-modules, where $\Gamma^{\prime} M$ (resp. $\left.\Gamma^{\prime} N\right)$ is a cell $R$-module. Then, according to [8, IV, 7.5], we have a commutative diagram

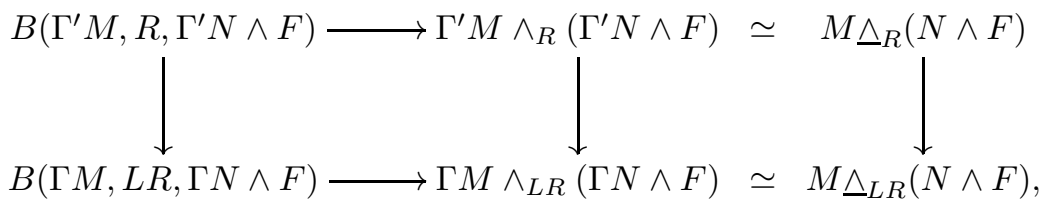

and the horizontal arrows are weak homotopy equivalences. Here $B(X, Y, Z)$ denotes the geometric realization of the simplicial $S^{0}$-module $B_{*}(X, Y, Z)$, the simplicial bar construction for a right $Y$-module $X$ and a left $Y$-module $Z$. Thus we need only show that the left vertical arrow is also a weak equivalence.

Since $R$ and $L R$ are $q$-cofibrant $S^{0}$-algebras, $B_{*}\left(\Gamma^{\prime} M, R, \Gamma^{\prime} N \wedge F\right)$ and $B_{*}(\Gamma M, L R, \Gamma N \wedge F)$ are proper simplicial $S^{0}$-modules [8, IV, 7.6], and it suffices to show that

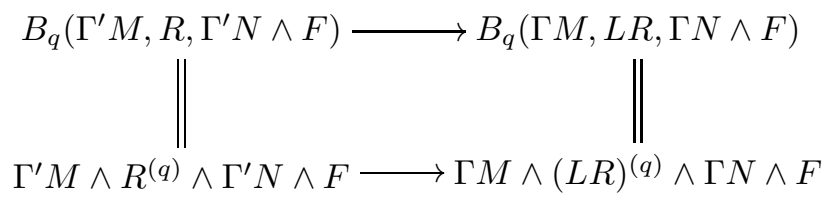

is a weak equivalence. The map

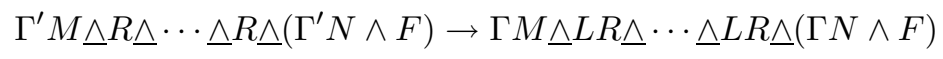


is certainly a weak equivalence; we thus only need to show that these derived smash products are represented by the point-set level smash products of Lemma 3.5. By Theorem 1.2, we are reduced to verifying that $\Gamma^{\prime} M, \Gamma M, R, L R, \Gamma^{\prime} N \wedge F$, and $\Gamma N \wedge F$ are all in $\overline{\mathcal{E}}_{S^{0}}$. But $R$ and $L R$ are in $\overline{\mathcal{E}}_{S^{0}}$ because they are $q$-cofibrant commutative $S^{0}$-algebras, and the others are in $\overline{\mathcal{E}}_{S^{0}}$ because they are either cell $R$-modules or cell $L R$-modules (see Proposition 1.4). This completes the proof.

We will use a Künneth type spectral sequence to compute

$$
\pi_{*} \widehat{L}\left[\Gamma^{j}\left(\widehat{L}\left(E^{h H} \wedge E\right) ; \widehat{L}\left(E^{h K} \wedge E\right)\right)\right] .
$$

This requires understanding the structure of $\pi_{*} \widehat{L}\left(E^{h H} \wedge E\right)=\operatorname{Map}_{c}\left(H \backslash G_{n}, E_{*}\right)$ as a module over $\pi_{*} \widehat{L}\left(E^{h K} \wedge E\right)=\operatorname{Map}_{c}\left(K \backslash G_{n}, E_{*}\right)$. It will be useful to proceed a little more generally.

Say that a commutative ring $C$ is a complete ring if it is provided with a complete decreasing filtration $\left\{F^{s} C\right\}_{s \geq 0}$ by ideals such that $F^{s} C \cdot F^{t} C \subset F^{s+t} C$ for all $s, t$. $C / F^{s} C$ is given the discrete topology, and $C=\lim _{\leftarrow s} C / F^{s} C$ is then given the topology of the inverse limit.

Lemma 3.14. Let $S$ and $T$ be profinite sets, and let $C$ be a complete commutative ring. Then there is a canonical isomorphism

$$
\operatorname{Map}_{c}(S, C) \widehat{\otimes}_{C} \operatorname{Map}_{c}(T, C) \rightarrow \operatorname{Map}_{c}(S \times T, C) .
$$

Proof. By definition,

$$
\operatorname{Map}_{c}(S, C) \widehat{\otimes}_{C} \operatorname{Map}_{c}(T, C)=\lim _{\leftarrow j} \operatorname{Map}_{c}(S, C) \otimes_{C} \operatorname{Map}_{c}(T, C) / F^{j},
$$

where

$$
F^{j}=\underset{s+t=j}{+} \operatorname{Map}_{c}\left(S, F^{s} C\right) \otimes_{C} \operatorname{Map}_{s}\left(T, F^{t} C\right) .
$$

Moreover, if $X$ is any profinite set,

$$
\operatorname{Map}_{c}(X, C)=\lim _{\leftarrow s} \operatorname{Map}_{c}\left(X, C / F^{s} C\right)=\lim _{\leftarrow s} \operatorname{Map}_{c}(X, C) / \operatorname{Map}_{c}\left(X, F^{s} C\right) .
$$

It therefore suffices to show that the canonical map

$$
\operatorname{Map}_{c}\left(S, C / F^{s} C\right) \otimes_{C} \operatorname{Map}_{c}\left(T, C / F^{s} C\right) \rightarrow \operatorname{Map}_{c}\left(S \times T, C / F^{s} C\right)
$$

is an isomorphism. But this follows by reduction to the case where $S$ and $T$ are finite.

Lemma 3.15. Let $G$ be a profinite group, and let $H$ and $K$ be closed subgroups of $G$ with $H$ normal in $K$. If $C$ is any complete commutative ring, then

$$
\operatorname{Map}_{c}(H \backslash G, C) \approx \operatorname{Map}_{c}(H \backslash K, C) \widehat{\otimes}_{C} \operatorname{Map}_{c}(K \backslash G, C)
$$

as $\operatorname{Map}_{c}(K \backslash G, C)$-algebras.

Proof. Since the above lemma implies that

$$
\operatorname{Map}_{c}(H \backslash K, C) \widehat{\otimes}_{C} \operatorname{Map}_{c}(K \backslash G, C)=\operatorname{Map}_{c}(H \backslash K \times K \backslash G, C),
$$

it suffices to show that there exists a homeomorphism $H \backslash K \times K \backslash G \rightarrow H \backslash G$ such that the diagram

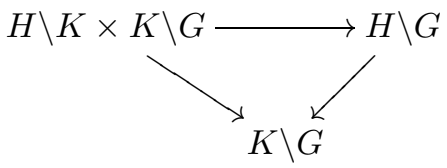


commutes. By [17, I $\S 1$, Proposition 1], there exists a continuous section $s: K \backslash G \rightarrow$ $H \backslash G$. Then define $H \backslash K \times K \backslash G \rightarrow H \backslash G$ by sending $(H x, K g)$ to $H x \cdot s(K g)$.

We can now make the desired computation of $\pi_{*} \widehat{L}\left[\Gamma^{j}\left(\widehat{L}\left(E^{h H} \wedge E\right) ; \widehat{L}\left(E^{h K} \wedge E\right)\right)\right]$.

Proposition 3.16. Let $M(I)$ be a generalized Toda $V(n-1)$ corresponding to the multi-index $\left(i_{0}, \ldots, i_{n-1}\right)$ as in the statement of Lemma 3.5. and let $H$ and $K$ be closed subgroups of $G_{n}$ with $H$ normal in $K$. Write $A=\pi_{*} \widehat{L}\left(E^{h K} \wedge E\right)$, $B=\pi_{*} \widehat{L}\left(E^{h H} \wedge E\right)$, and $J=\left(p^{i_{0}}, v_{1}^{i_{1}}, \ldots, v_{n-1}^{i_{n-1}}\right) \subset E_{*}$. Then

$$
B \otimes_{A} \cdots \otimes_{A} B / J\left(B \otimes_{A} \cdots \otimes_{A} B\right) \stackrel{\approx}{\rightarrow} \pi_{*}\left[\Gamma^{j}\left(\widehat{L}\left(E^{h H} \wedge E\right) ; \widehat{L}\left(E^{h K} \wedge E\right)\right) \wedge M(I)\right] .
$$

Proof. We will prove this by induction on $j$, the result being trivial for $j=0$. Begin by assuming that

$$
\underbrace{B \widehat{\otimes}_{A} \cdots \widehat{\otimes}_{A} B}_{j \text { copies }} \stackrel{\approx}{\rightarrow} \pi_{*} \widehat{L}\left[\Gamma^{j-1}\left(\widehat{L}\left(E^{h H} \wedge E\right) ; \widehat{L}\left(E^{h K} \wedge E\right)\right)\right],
$$

where the completed tensor product is with respect to the $J$-adic filtration on $B$. Now

$$
B \widehat{\otimes}_{A} \cdots \widehat{\otimes}_{A} B=\operatorname{Map}_{c}(\underbrace{H \backslash G_{n} \times_{K \backslash G_{n}} \cdots \times_{K \backslash G_{n}} H \backslash G_{n}}_{j \text { copies }}, E_{*})
$$

(cf. Lemma 3.14 ); in particular, $B \widehat{\otimes}_{A} \cdots \widehat{\otimes}_{A} B$ is flat over $E_{*}$.

Write $N=B \widehat{\otimes}_{A} \cdots \widehat{\otimes}_{A} B$. By [8, IV, Theorem 4.1], there is a spectral sequence $\operatorname{Tor}_{s, t}^{A}\left(N, \pi_{*}\left[\widehat{L}\left(E^{h H} \wedge E\right) \wedge M(I)\right]\right) \Rightarrow \pi_{s+t}\left[\Gamma^{j}\left(\widehat{L}\left(E^{h H} \wedge E\right) ; \widehat{L}\left(E^{h K} \wedge E\right)\right) \wedge M(I)\right]$, so we need only show that

$$
\operatorname{Tor}_{s, *}^{A}\left(N, \operatorname{Map}_{c}\left(H \backslash G_{n}, E_{*} / J\right)\right)=0
$$

for all $s>0 . N$ and $A$ are both flat over $E_{*}$; therefore

$$
\operatorname{Tor}_{s, *}^{A}\left(N, \operatorname{Map}_{c}\left(H \backslash G_{n}, E_{*} / J\right)\right)=\operatorname{Tor}_{s, *}^{A / J A}\left(N / J N, \operatorname{Map}_{c}\left(H \backslash G_{n}, E_{*} / J\right)\right) .
$$

Moreover, by the preceding lemma,

$$
\operatorname{Map}_{c}\left(H \backslash G_{n}, E_{*} / J\right) \approx \operatorname{Map}_{c}\left(K / H, E_{*} / J\right) \otimes_{E_{*} / J} A / J A .
$$

Since $\operatorname{Map}_{c}\left(K / H, E_{*} / J\right)$ is flat over $E_{*} / J$, we then have

$$
\begin{aligned}
\operatorname{Tor}_{s, *}^{A / J A}\left(N / J N, \operatorname{Map}_{c}\left(H \backslash G_{n}, E_{*} / J\right)\right) & =\operatorname{Tor}_{s, *}^{E_{*} / J}\left(N / J N, \operatorname{Map}_{c}\left(K / H, E_{*} / J\right)\right) \\
& =0
\end{aligned}
$$

whenever $s>0$. This completes the induction and the proof.

With the computation of $\pi_{*} \widehat{L}\left(I^{j} \underline{\triangle} E\right)$ in hand, we can now begin the proof of Theorem 3.7. We start by considering a special case; namely we assume that $K / H$ is finite. Define an $E^{h K}$-module map

$$
\tau_{j}: I^{j} \rightarrow \bigvee_{(K / H)^{j}} E^{h H}
$$

by requiring that the projection onto the summand indexed by $\left(\bar{g}_{1}, \ldots, \bar{g}_{j}\right)$ be given by the composition

$$
I^{j} \stackrel{\widehat{L}\left(\bar{g}_{1}^{-1} \wedge \cdots \wedge \bar{g}_{j}^{-1} \wedge E^{h H}\right)}{\longrightarrow} I^{j} \stackrel{\mu}{\longrightarrow} E^{h H} .
$$

The next result implies immediately that $T_{j}$ is an isomorphism in this case. 
Proposition 3.17. If $K$ and $H$ are closed subgroups of $G_{n}$ with $H$ normal in $K$ and $K / H$ finite, then $\tau_{j}$ is a weak equivalence.

Proof. First assume that $K$ and $H$ are open in $G_{n}$. We will prove that

$$
\begin{aligned}
\widehat{L}\left(\tau_{j} \wedge E\right)_{*}: \pi_{*} \widehat{L}\left(I^{j} \wedge E\right) \longrightarrow & \pi_{*} \underset{(K / H)^{j}}{\bigvee} \widehat{L}\left(E^{h H} \wedge E\right) \\
& \bigoplus_{(K / H)^{j}} \operatorname{Map}\left(H \backslash G_{n}, E_{*}\right)
\end{aligned}
$$

is an isomorphism. By Lemma 3.12 and Proposition 3.16

$\pi_{*} \widehat{L}\left(I^{j} \triangle E\right)=\operatorname{Map}\left(H \backslash G_{n}, E_{*}\right) \otimes_{\operatorname{Map}\left(K \backslash G_{n}, E_{*}\right)} \cdots \otimes_{\operatorname{Map}\left(K \backslash G_{n}, E_{*}\right)} \operatorname{Map}\left(H \backslash G_{n}, E_{*}\right)$.

Tracking down the identifications, it follows from (3.2) that the projection $p_{\left(\bar{g}_{1}, \ldots, \bar{g}_{j}\right)}$ of $\widehat{L}\left(\tau_{j} \wedge E\right)_{*}$ onto the summand indexed by $\left(\bar{g}_{1}, \ldots, \bar{g}_{j}\right) \in(K / H)^{j}$ is given by

$$
p_{\left(\bar{g}_{1}, \ldots, \bar{g}_{j}\right)}\left(f_{1}, \ldots, f_{j+1_{1}}\right)(H g)=f_{1}\left(\bar{g}_{1} g\right) f_{2}\left(\bar{g}_{2} g\right) \cdots f_{j}\left(\bar{g}_{j} g\right) f_{j+1}(H g) .
$$

This formula also defines a map

$$
\begin{aligned}
z_{j}: \operatorname{Map}\left(H \backslash G_{n}, \mathbb{Z}_{p}\right) \otimes_{\operatorname{Map}\left(K \backslash G_{n}, \mathbb{Z}_{p}\right)} \cdots \otimes_{\operatorname{Map}\left(K \backslash G_{n}, \mathbb{Z}_{p}\right)} & \operatorname{Map}\left(H \backslash G_{n}, \mathbb{Z}_{p}\right) \\
& \rightarrow \bigoplus_{(K / H)^{j}} \operatorname{Map}\left(H \backslash G_{n}, \mathbb{Z}_{p}\right)
\end{aligned}
$$

and $z_{j} \otimes E_{*}=\widehat{L}\left(\tau_{j} \wedge E\right)_{*} . z_{j}$ is easily seen to be an epimorphism; we claim that this implies that $z_{j}$ is in fact an isomorphism. Indeed, by Lemma 3.15.

$$
\operatorname{Map}\left(H \backslash G_{n}, \mathbb{Z}_{p}\right)=\operatorname{Map}\left(K / H, \mathbb{Z}_{p}\right) \otimes_{\mathbb{Z}_{p}} \operatorname{Map}\left(K \backslash G_{n}, \mathbb{Z}_{p}\right),
$$

so $z_{j}$ becomes a map

$$
\begin{aligned}
\underbrace{\operatorname{Map}\left(K / H, \mathbb{Z}_{p}\right) \otimes_{\mathbb{Z}_{p}} \cdots \otimes_{\mathbb{Z}_{p}} \operatorname{Map}\left(K / H, \mathbb{Z}_{p}\right)}_{j+1 \text { copies }} \otimes_{\mathbb{Z}_{p}} \operatorname{Map}\left(K \backslash G_{n}, \mathbb{Z}_{p}\right) \\
\rightarrow \bigoplus_{(K / H)^{j}} \operatorname{Map}\left(H \backslash G_{n}, \mathbb{Z}_{p}\right) .
\end{aligned}
$$

Both sides are free $\mathbb{Z}_{p}$-modules of the same finite rank and therefore $z_{j}$ must be a monomorphism as well.

For the general case, recall the sequence $\left\{U_{i}\right\}$ of open normal subgroups of (3.1), and let $H_{i}=H U_{i}, K_{i}=K U_{i}$. Then

$$
\widehat{L}\left(\underset{\rightarrow i}{\operatorname{holim}} \Gamma^{j}\left(E^{h H_{i}} ; E^{h K_{i}}\right)\right) \stackrel{\simeq}{\rightrightarrows} \widehat{L}\left(\Gamma^{j}\left(E^{h H} ; E^{h K}\right)\right) .
$$

There are several ways to see this. The most painless is to use Proposition 3.16 to verify that

$$
\pi_{*} \widehat{L}\left(\underset{\rightarrow i}{\operatorname{holim}} \Gamma^{j}\left(E^{h H_{i}} ; E^{h K_{i}}\right) \triangle E\right) \underset{\rightarrow}{\approx} \pi_{*} \widehat{L}\left(\Gamma^{j}\left(E^{h H} ; E^{h K}\right) \triangle E\right) .
$$

Alternatively, one can use techniques such as Proposition 1.4 to replace derived smash products by ordinary smash products and then use their good properties with respect to colimits. Lemma 3.13 will also be involved at the end.

We have already proved that

$$
\tau_{j}: \widehat{L} \Gamma^{j}\left(E^{h H_{i}} ; E^{h K_{i}}\right) \rightarrow \bigvee_{\left(K_{i} / H_{i}\right)^{j}} E^{h H_{i}}
$$


is an equivalence; the result in general now follows from (3.7), the naturality of $\tau_{j}$, and the fact that $K / H \rightarrow K_{i} / H_{i}$ is a bijection for $i$ sufficiently large.

Proof of Theorem 3.7. First observe that it suffices to show that

$$
T_{j}:\left[X_{\alpha}, I^{j} \wedge M(I)\right]_{*}^{E(K)} \rightarrow \operatorname{Map}_{c}\left((K / H)^{j},\left[X_{\alpha}, E^{h H} \wedge M(I)\right]_{*}^{E(K)}\right)
$$

is an isomorphism for any finite cell $E^{h K}$-module $X_{\alpha}$ and multi-index $I$. Then, since $\left[X_{\alpha}, E^{h H} \wedge M(I)\right]_{*}^{E(K)}$ is finite in each degree (Lemma 3.5) and $\operatorname{Map}_{c}\left((K / H)^{j}, ?\right)$ is exact on the category of profinite abelian groups (see [17, I§1, Proposition 1]), all the relevant $\lim ^{i}$ terms vanish, and we have

$$
\begin{aligned}
{\left[X, I^{j}\right]_{*}^{E(K)} } & =\lim _{\leftarrow}\left[X_{\alpha}, I^{j} \wedge M(I)\right]_{*}^{E(K)}, \\
\operatorname{Map}_{c}\left((K / H)^{j},\left[X, E^{h H}\right]_{*}^{E(K)}\right) & =\lim _{\leftarrow} \operatorname{Map}_{c}\left((K / H)^{j},\left[X_{\alpha}, E^{h H} \wedge M(I)\right]_{*}^{E(K)}\right) .
\end{aligned}
$$

Let $\left\{U_{i}\right\}$ be as in the previous proof, and let $H_{i}=K \cap H U_{i}$. Then $K / H_{i}$ is finite, so from above,

$$
T_{j}:\left[X_{\alpha}, \Gamma^{j}\left(E^{h H_{i}} ; E^{h K}\right) \wedge M(I)\right]_{*}^{E(K)} \underset{\sim}{\rightarrow} \operatorname{Map}\left(\left(K / H_{i}\right)^{j},\left[X_{\alpha}, E^{h H_{i}} \wedge M(I)\right]_{*}^{E(K)}\right) .
$$

But, as in the proof above,

$$
\underset{\rightarrow i}{\operatorname{holim}} \Gamma^{j}\left(E^{h H_{i}} ; E^{h K}\right) \wedge M(I) \rightarrow \Gamma^{j}\left(E^{h H} ; E^{h K}\right) \wedge M(I)=I^{j} \wedge M(I)
$$

is an equivalence in $\mathcal{D}_{E(K)}$; thus

$$
\left[X_{\alpha}, I^{j} \wedge M(I)\right]_{*}^{E(K)}=\lim _{\rightarrow i}\left[X_{\alpha}, \Gamma^{j}\left(E^{h H_{i}} ; E^{h K}\right) \wedge M(I)\right]_{*}^{E(K)} .
$$

Moreover,

$\operatorname{Map}_{c}\left((K / H)^{j},\left[X_{\alpha}, E^{h H} \wedge M(I)\right]_{*}^{E(K)}\right)=\lim _{\rightarrow i} \operatorname{Map}\left(\left(K / H_{i}\right)^{j},\left[X_{\alpha}, E^{h H_{i}} \wedge M(I)\right]_{*}^{E(K)}\right)$, and again it follows from the naturality of $T_{j}$ that the map in (3.8) is an isomorphism.

\section{Convergence}

Our proof of Theorem 3.3 will involve two main steps. We will first prove that $E^{h K}$ is $\widehat{L}-E_{*}^{E(K)}$ local. We will then establish a vanishing line in the E-Adams spectral sequence in $\widehat{L} \mathcal{D}_{E(K)}$; by virtue of Proposition 2.5 this implies that $E^{h K}$ is $E$-nilpotent in $\widehat{L} \mathcal{D}_{E(K)}$. But $E$ is an $E^{h H}$-module in $\mathcal{D}_{E(K)}$; therefore $E$ is $E^{h H_{-}}$ nilpotent in $\widehat{L} \mathcal{D}_{E(K)}$, and hence so is $E^{h K}$. This immediately implies the conclusion of Theorem 3.3

The proof of the first step requires an analysis of $\widehat{L}\left(E \wedge E^{h K}\right)$. Recall that $E$ and $E^{h K}$ (and hence $E^{h K} \wedge E$ and $E \wedge E$ ) are cell commutative $S^{0}$-algebras; thus the maps

$$
\begin{aligned}
& E^{h K}=E^{h K} \wedge S^{0} \rightarrow E^{h K} \wedge E \rightarrow \widehat{L}\left(E^{h K} \wedge E\right), \\
& E^{h K}=E^{h K} \wedge S^{0} \rightarrow E \wedge E \rightarrow \widehat{L}(E \wedge E)
\end{aligned}
$$

are algebra maps. In particular, these maps give $\widehat{L}\left(E^{h K} \wedge E\right)$ and $\widehat{L}(E \wedge E)$ the structure of $E^{h K}$-modules.

Lemma 4.1. $\widehat{L}\left(E^{h K} \wedge E\right)$ is a retract of $\widehat{L}(E \wedge E)$ in $\mathcal{D}_{E(K)}$. 
Proof. We will prove that $\widehat{L}\left(E^{h K} \wedge E\right)$ is a retract of $\widehat{L}(E \wedge E)$ in $\mathcal{D}_{\widehat{L}(E(K) \wedge E)}$; that is, there exists a map $r: \widehat{L}(E \wedge E) \rightarrow \widehat{L}\left(E^{h K} \wedge E\right)$ in $\mathcal{D}_{\widehat{L}(E(K) \wedge E)}$ such that the composition

$$
\widehat{L}\left(E^{h K} \wedge E\right) \rightarrow \widehat{L}(E \wedge E) \stackrel{r}{\rightarrow} \widehat{L}\left(E^{h K} \wedge E\right)
$$

is a weak equivalence. The desired result then follows upon applying the functor $\mathcal{D}_{\widehat{L}(E(K) \wedge E)} \rightarrow \mathcal{D}_{E(K)}$.

First observe that there is a retraction

$$
h: \pi_{*} \widehat{L}(E \wedge E) \rightarrow \pi_{*} \widehat{L}\left(E^{h K} \wedge E\right)
$$

of $\pi_{*} \widehat{L}\left(E^{h K} \wedge E\right)$ modules. Indeed, let $h$ be the map

$$
\pi_{*} \widehat{L}(E \wedge E)=\operatorname{Map}_{c}\left(G_{n}, E_{*}\right) \stackrel{\operatorname{Map}_{c}\left(s, E_{*}\right)}{\longrightarrow} \operatorname{Map}_{c}\left(K \backslash G_{n}, E_{*}\right)=\pi_{*} \widehat{L}\left(E^{h K} \wedge E\right),
$$

where $s: K \backslash G_{n} \rightarrow G_{n}$ is a continuous cross-section of the projection $G_{n} \rightarrow K \backslash G_{n}$ ([17, I§1, Proposition 1]). It is clear that $h$ is a retraction; we thus only need to show that it is a map of $\pi_{*} \widehat{L}\left(E^{h K} \wedge E\right)$-modules. This amounts to showing that the diagram

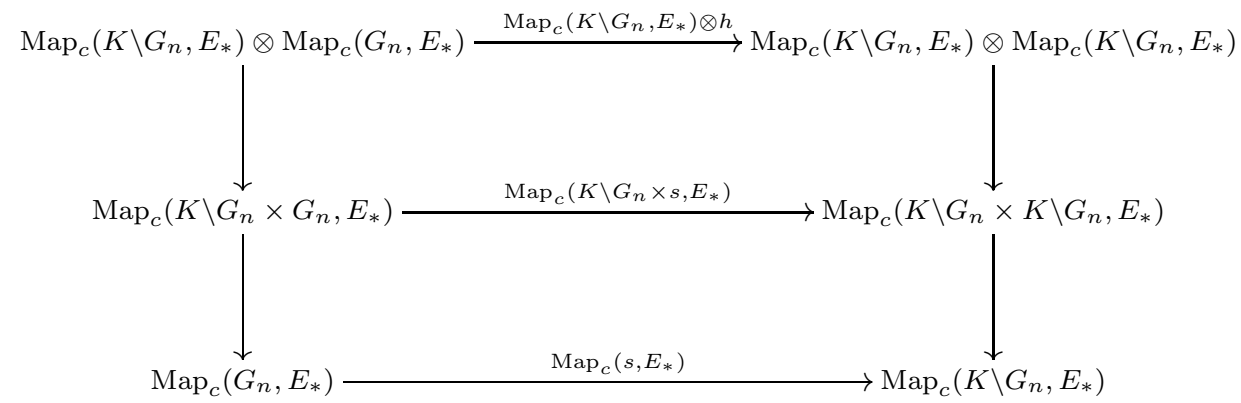

commutes, where the lower vertical maps are induced by diagonal maps. But this is easy to check.

Now write $A=\pi_{*} \widehat{L}\left(E^{h K} \wedge E\right)$ and $B=\pi_{*} \widehat{L}(E \wedge E)$. There is a spectral sequence

$$
\operatorname{Ext}_{A}^{* *}(B, A / J A) \Rightarrow\left[\widehat{L}(E \wedge E), \widehat{L}\left(E^{h K} \wedge E \wedge M(I)\right]_{*}^{\widehat{L}(E(K) \wedge E)}\right.
$$

[8, IV, Theorem 4.1]. As usual, $I$ is the multi-index $\left(i_{0}, \ldots, i_{n-1}\right)$, and $J$ is the ideal $\left(p^{i_{0}}, v_{1}^{i_{1}}, \ldots, v_{n-1}^{i_{n-1}}\right)$ in $E_{*}$. We will show that $\operatorname{Ext}_{A}^{s, *}(B, A / J A)=0$ for all $s>0$; this implies that there is a retraction $r$ in $\mathcal{D}_{\widehat{L}(E(K) \wedge E)}$ with $\pi_{*} r=h$.

Since $B$ and $A$ are both flat over $E_{*}$,

$$
\operatorname{Ext}_{A}^{* *}(B, A / J A)=\operatorname{Ext}_{A / J A}(B / J B, A / J A) .
$$

By Lemma 3.15 with $H=\{e\}$,

$$
B / J B \approx \operatorname{Map}_{c}\left(K, E_{*} / J\right) \otimes_{E_{*} / J} A / J A
$$

as $A / J A$-algebras. Since $\operatorname{Map}_{c}\left(K, E_{*} / J\right)$ is a flat $E_{*} / J$-module, it then follows that

$$
\operatorname{Ext}_{A / J A}^{* *}(B / J B, A / J A)=\operatorname{Ext}_{E_{*} / J}^{* *}\left(\operatorname{Map}_{c}\left(K, E_{*} / J\right), A / J A\right)
$$

and that

$$
\operatorname{Ext}_{E_{*} / J}^{* *}\left(\operatorname{Map}_{c}\left(K, E_{*} / J\right), N\right)=\operatorname{Ext}_{\mathbb{F}_{p^{n}\left[u, u^{-1}\right]}^{* *}}\left(\operatorname{Map}_{c}\left(K, E_{*} / I_{n}\right), N\right)
$$

whenever $N$ is an $E_{*}$-module annihilated by the ideal $I_{n}=\left(p, v_{1}, \ldots, v_{n-1}\right)$. Hence

$$
\operatorname{Ext}_{E_{*} / J}^{s, *}\left(\operatorname{Map}_{c}\left(K, E_{*} / J\right), N\right)=0
$$


for all $s>0$. Now consider the short exact sequences

$$
0 \rightarrow \frac{\left(I_{n}^{k}+J\right) A}{\left(I_{n}^{k+1}+J\right) A} \rightarrow \frac{A}{\left(I_{n}^{k+1}+J\right) A} \rightarrow \frac{A}{\left(I_{n}^{k}+J\right) A} \rightarrow 0
$$

and take $N=\left(I_{n}^{k}+J\right) A /\left(I_{n}^{k+1}+J\right) A$. Using induction on $k$, we obtain that

$$
\operatorname{Ext}_{E_{*} / J}^{s, *}\left(\operatorname{Map}_{c}\left(K, E_{*} / J\right), A /\left(I_{n}^{k}+J\right) A\right)=0
$$

for all $s>0$ and $k \geq 0$. But $I_{n}^{k} \subset J$ for $k$ sufficiently large; therefore

$$
\operatorname{Ext}_{A}^{s, *}(B, A / J A)=\operatorname{Ext}_{E_{*} / J}^{s, *}\left(\operatorname{Map}_{c}\left(K, E_{*} / J\right), A / J A\right)=0
$$

whenever $s>0$.

Lemma 4.2. $E^{h K}$ is $\widehat{L}-E_{*}^{E(K)}$ local.

Proof. Let $X$ be a cell $E^{h K}$-module. We must show that if $X \wedge_{E(K)}(E \wedge K(n)) \simeq *$ in $\mathcal{D}_{E(K)}$, then $\left[X, E^{h K}\right]_{*}^{E(K)}=0$. As usual, we take $K(n)$ to be a cell $S^{0}$-module.

Let $\Gamma E$ be a cell $E^{h K}$-module and $\Gamma E \rightarrow E$ a weak equivalence of $E^{h K}$-modules. Then $X \wedge_{E(K)}(E \wedge K(n))$ is trivial in $\mathcal{D}_{E(K)}$ if and only if $\left(X \wedge_{E(K)} \Gamma E\right) \wedge K(n)$ is contractible in $\mathcal{M}_{E(K)}$. This implies that $\left[X \wedge_{E(K)}(\Gamma E \wedge E)\right] \wedge K(n)$ is contractible, where $E^{h K}$ acts on the left factor of $\Gamma E \wedge E$. But $\Gamma E \wedge E \rightarrow E \wedge E$ is a weak equivalence since $E$ is a $q$-cofibrant commutative $S^{0}$-algebra and $\Gamma E$ is a cell module over a $q$-cofibrant commutative $S^{0}$-algebra (see Theorem 1.2 Proposition 1.4). Hence $\left[X \wedge_{E(K)}(E \wedge E)\right] \wedge K(n)$, and therefore $\left[X \wedge_{E(K)} \widehat{L}(E \wedge E)\right] \wedge K(n)$, is weakly contractible. (Here we have applied Proposition 1.1 a number of times.) By the preceding lemma, it follows that $\left(X \wedge_{E(K)} \widehat{L}\left(E^{h K} \wedge E\right)\right) \wedge K(n)$ is also weakly contractible. Thus

$$
\left[X \wedge_{E(K)}\left(E^{h K} \wedge E\right)\right] \wedge K(n)=X \wedge E \wedge K(n)
$$

is weakly contractible. Now apply Propositions 1.11.4 again to obtain that $X \wedge E \wedge K(n)$ is weakly equivalent to $X \wedge E \wedge K(n)$; since $E \wedge K(n)$ is a wedge of $K(n)^{\prime}$ 's, this implies that $X$ is $K(n)_{*}$-acyclic. But $E^{h K}$ is $K(n)_{*}$-local, and the $K(n)_{*}$-localization of an $E^{h K}$-module is the same as the $\left(E^{h K} \wedge K(n)\right)_{*}^{E(K)}$ localization; therefore $\left[X, E^{h K}\right]_{*}^{E(K)}=0$. This completes the proof.

The next result provides us with the requisite vanishing line.

Lemma 4.3. Let $K$ be a closed subgroup of $G_{n}$, let $P$ be a p-Sylow subgroup of $K$, and let $X$ be the $p$-localization of a finite spectrum with free $\mathbb{Z}_{(p)}$-homology such that

$$
H_{c}^{s, *}\left(P, E_{*} X / I_{n} E_{*} X\right)=0
$$

for all $s$ bigger than some $s_{0}$. Then, if $Z$ is any $E^{h K}$-module,

$$
H_{c}^{s, *}\left(K,[Z, E \wedge X]_{E(K)}^{*}\right)=0
$$

for all $s>s_{0}$.

Remark 4.4. The topology on $[Z, E \wedge X]_{E(K)}^{*}=[Z \wedge D X, E]_{E(K)}^{*}$ is just that of Proposition 3.6 with $H=\{e\}$.

Once this lemma is proved, we easily obtain the following result, which, by the remarks at the beginning of this section, implies Theorem 3.3 .

Lemma 4.5. $E^{h K}$ is E-nilpotent in $\widehat{L} \mathcal{D}_{E(K)}$. 
Proof. By the discussion in [6] proof of Theorem 5.3], there exists an $X$ satisfying the hypotheses of the previous lemma. It then follows from Proposition [2.5 that $E^{h K} \wedge X$ is $E$-nilpotent in $\widehat{L} \mathcal{D}_{E(K)}$.

Now consider the class $\mathcal{N}$ consisting of all finite $p$-local spectra $Y$ such that $E^{h K} \wedge Y$ is $E$-nilpotent in $\widehat{L} \mathcal{D}_{E(K)}$. Nilpotence technology [13, Theorem 7] tells us that $\mathcal{N}$ is the collection of $K(m-1)_{*}$-acyclic spectra for some $m \geq 0$. Since $X \in \mathcal{N}$, we must have that $\mathcal{N}$ consists of all finite $p$-local spectra. In particular, $S_{(p)}^{0} \in \mathcal{N}$ and thus $E^{h K}$ is $E$-nilpotent in $\widehat{L} \mathcal{D}_{E(K)}$.

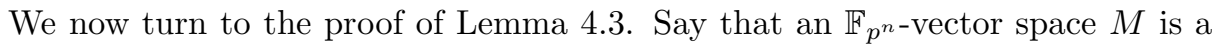
twisted $K$-module over $\mathbb{F}_{p^{n}}$ if it is a $\mathbb{Z}[K]$-module such that

$$
g(\mathrm{~cm})=(\pi(\mathrm{g}) \mathrm{c}) \cdot \mathrm{gm}
$$

for all $g \in K, c \in \mathbb{F}_{p^{n}}$, and $m \in M$, where $\pi: K \rightarrow$ Gal is the usual projection. We then have the following preliminary result. Our notation is as in Lemma 4.3 .

Lemma 4.6. Suppose that $N$ is a finite discrete twisted $K$-module over $\mathbb{F}_{p^{n}}$ with the property that $H_{c}^{s}(P, N)=0$ for all s bigger than some $s_{0}$. Then $H_{c}^{s}\left(K, M \otimes_{\mathbb{F}_{p^{n}}} N\right)=$ 0 for all $s>s_{0}$ whenever $M$ is a finite discrete twisted $K$-module over $\mathbb{F}_{p^{n}}$ and $K$ acts diagonally on $M \otimes_{\mathbb{F}_{p^{n}}} N$.

Proof.

$$
H_{c}^{*}\left(K, M \otimes_{\mathbb{F}_{p^{n}}} N\right) \rightarrow H_{c}^{*}\left(P, M \otimes_{\mathbb{F}_{p^{n}}} N\right)
$$

is a monomorphism, so we need only show that $H_{c}^{s}\left(P, M \otimes_{\mathbb{F}_{p^{n}}} N\right)=0$ whenever $s>s_{0}$.

Let $L$ be the image of the projection $\pi: P \rightarrow \mathrm{Gal}$, so that $L=\operatorname{Gal}\left(\mathbb{F}_{p^{n}} / \mathbb{F}_{p^{m}}\right)$ for some $m \geq 1$, and let $P_{0}$ be the kernel of $\pi$. Since $P_{0}$ is $p$-analytic, $H_{c}^{j}\left(P_{0}, M \otimes_{\mathbb{F}_{p}} N\right)$

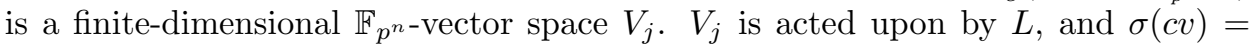
$\sigma(c) \sigma(v)$ for all $\sigma \in L, c \in \mathbb{F}_{p^{n}}$, and $v \in V_{j}$. It then follows (cf. [5, Lemma 5.4]) that $V_{j}=V_{j}^{L} \otimes_{\mathbb{F}_{p} m} \mathbb{F}_{p^{n}}$ as $\mathbb{F}_{p^{n} \text {-vector spaces and as } L \text {-modules, so that }}$

$$
H^{i}\left(L, H_{c}^{j}\left(P_{0}, M \otimes_{\mathbb{F}_{p^{n}}} N\right)\right)=\left\{\begin{array}{cc}
H_{c}^{j}\left(P_{0}, M \otimes_{\mathbb{F}_{p^{n}}} N\right)^{L} & i=0, \\
0 & i \neq 0 .
\end{array}\right.
$$

Therefore, by the Lyndon-Hochschild-Serre spectral sequence for profinite groups (see [18, II, §4]),

$$
H_{c}^{s}\left(P, M \otimes_{\mathbb{F}_{p^{n}}} N\right)=0 \Leftrightarrow H_{c}^{s}\left(P_{0}, M \otimes_{\mathbb{F}_{p^{n}}} N\right)=0 .
$$

The point here is that $M$ and $N$ are both $P_{0}$-modules over $\mathbb{F}_{p^{n}}$; there is no Galois action on $\mathbb{F}_{p^{n}}$ to worry about.

Since $P_{0}$ is a profinite $p$-group, the only finite simple discrete $P_{0}$-module over $\mathbb{F}_{p^{n}}$ is $\mathbb{F}_{p^{n}}$ with the trivial action (cf. [18, Propositon 17]). By considering a composition series for $M$, it now follows easily that $H_{c}^{s}\left(P_{0}, M \otimes_{\mathbb{F}_{p^{n}}} N\right)=0$ whenever $s>s_{0}$. This completes the proof.

Proof of Lemma 4.3. By Proposition 3.6 and the definition of cohomology, it suffices to prove that

$$
H_{c}^{s, *}\left(K,[Z \wedge M(I), E \wedge X]_{E(K)}^{*}\right)=0
$$

for all $s>s_{0}$ and multi-indices $I$, whenever $Z$ is a finite cell $E^{h K}$-module.

Consider now the map

$$
E_{E(K)}^{*}(Z \wedge M(I)) \otimes_{E_{*}} E_{*} X \rightarrow[Z \wedge M(I), E \wedge X]_{E(K)}^{*}
$$


which sends $f \otimes c$ to the composition

$$
Z \wedge M(I)=Z \wedge M(I) \wedge S^{0} \stackrel{f \wedge c}{\longrightarrow} E \wedge E \wedge X \stackrel{\mu \wedge X}{\longrightarrow} E \wedge X .
$$

Using induction on the $E^{h K}$-cells of $Z \wedge M(I)$, together with the fact that $E_{*} X$ is a free $E_{*}$-module, it is easy to check that this map is an isomorphism. Moreover, $E_{E(K)}^{*}(Z \wedge M(I))$ is annihilated by a finite power of $I_{n}$; it therefore suffices to show that

$$
H_{c}^{s, *}\left(K, I_{n}^{k} E_{E(K)}^{*}(Z \wedge M(I)) / I_{n}^{k+1} E_{E(K)}^{*}(Z \wedge M(I)) \otimes_{E_{*} / I_{n}} E_{*} X / I_{n} E_{*} X\right)=0
$$

for all $s>s_{0}$ and $k \geq 0$. But this follows from Lemma 4.6.

\section{Appendix A. A COnsistency Result}

Let $R$ be a commutative $S^{0}$-algebra, and let $X$ be an $R$-module on which a finite group $G$ acts by $R$-module maps. This action defines an evident functor from the category with one object and morphism group $G$ to the category of $R$-modules; we write $\Pi^{*} X$ for the cosimplicial replacement of this diagram. (Here, and in what follows, we use the terminology and notation of [4, Chapters $\mathrm{X}$ and XI].) $\operatorname{Tot}\left(\Pi^{*} X\right)=X^{h G}$, and the homotopy fixed point spectral sequence converging to $\left[Z, X^{h G}\right]_{R}^{*}$ is obtained by mapping $Z$ into the tower of fibrations $\left\{\operatorname{Tot}_{k} \Pi^{*} X\right\}$. If $K$ and $H$ are closed subgroups of $G_{n}$ with $H$ normal in $K$ and $K / H$ finite, then we may specialize to the case where $R=E^{h K}, X=E^{h H}$, and $G=K / H$.

Theorem A.1. With the notation as above, the homotopy fixed point spectral sequence agrees with the Adams spectral sequence $E_{r}^{*, *}\left(Z, E^{h K} ; E^{h H}\right)$ in $\widehat{L} \mathcal{D}_{E(K)}$.

Proof. Let $F_{k}$ denote the fiber of $\operatorname{Tot}_{k}\left(\Pi^{*} E^{h H}\right) \rightarrow \operatorname{Tot}_{k-1}\left(\Pi^{*} E^{h H}\right)$. It suffices to prove that the sequence

$$
* \rightarrow E^{h K} \rightarrow F_{0} \rightarrow \Sigma F_{1} \rightarrow \cdots
$$

is an $E^{h H}$-Adams resolution of $E^{h K}$ in $\widehat{L} \mathcal{D}_{E(K)}$. Each $F_{k}$ is a product of suspensions of copies of $E^{h H}$ and so is $E^{h H}$-injective in $\widehat{L} \mathcal{D}_{E(K)}$. We are therefore reduced to showing that

$$
0 \rightarrow\left[Z, \widehat{L}\left(E^{h K} \underline{\triangle}_{E(K)} E^{h H}\right]_{*}^{E(K)} \rightarrow\left[Z, \widehat{L}\left(F_{0} \Lambda_{E(K)} E^{h H}\right]_{*}^{E(K)} \rightarrow \cdots\right.\right.
$$

is exact for all cell $E^{h K}$-modules $Z$. But $\widehat{L}\left(F_{k} \Lambda_{E(K)} E^{h H}\right)$ is equivalent to the fiber of

$$
\operatorname{Tot}_{k}\left(\Pi^{*} \widehat{L}\left(E^{h H} \triangle_{E(K)} E^{h H}\right)\right) \rightarrow \operatorname{Tot}_{k-1}\left(\Pi^{*} \widehat{L}\left(E^{h H} \underline{\Lambda}_{E(K)} E^{h H}\right)\right),
$$

where $K / H$ acts on the left factor of $\widehat{L}\left(E^{h H} \triangle_{E(K)} E^{h H}\right)$. Hence

$$
H^{*}\left[Z, \widehat{L}\left(F_{*} \underline{\Lambda}_{E(K)} E^{h H}\right)\right]_{E(K)}^{*}=H^{*}\left(K / H,\left[Z, \widehat{L}\left(E^{h H} \underline{\triangle}_{E(K)} E^{h H}\right)\right]_{E(K)}^{*}\right) .
$$

By Corollary 3.9 ,

$$
\left[Z, \widehat{L}\left(E^{h H} \triangle_{E(K)} E^{h H}\right)\right]_{E(K)}^{*}=\operatorname{Map}_{c}\left(K / H,\left[Z, E^{h H}\right]_{E(K)}^{*}\right)
$$

as $K / H$-modules, and therefore

$$
H^{*}\left(K / H,\left[Z, \widehat{L}\left(E^{h H} \triangle_{E(K)} E^{h H}\right)\right]_{E(K)}^{i}\right)=\left[Z, E^{h H}\right]_{E(K)}^{i}
$$

concentrated in homological degree 0 . This proves that the desired sequence is exact. 
Remark A.2. The alert reader may have noticed that $\widehat{L}\left(E^{h H} \triangle_{E(K)} E^{h H}\right)$ in (A.2) is only a homotopy object and therefore the action of $K / H$ is only an action up to homotopy. However, an honest action may be constructed by observing that $E^{h H} \triangle_{E(K)} E^{h H}$ is equivalent to $E^{h H} \wedge_{E(K)} \Gamma E^{h H}$, where $\Gamma E^{h H}$ is a cell $E^{h K}$ module weakly equivalent to $E^{h H}$, and that $\widehat{L}$ can be constructed to be natural on $\mathcal{M}_{E(K)}$ (see [8, Chapter VIII]).

\section{REFERENCES}

[1] A. Baker and A. Lazerev, On the Adams spectral sequence for R-modules, Algebr. Geom. Topol. 1 (2001), 173-199 (electronic). MR 2002a:55011

[2] J. M. Boardman, Conditionally convergent spectral sequences, Homotopy Invariant Algebraic Structures (J. P. Meyer, J. Morava, W. S. Wilson, eds.), Contemp. Math. 239, Amer. Math. Soc., Providence, RI, 1999, pp. 49-84. MR 2000m:55024

[3] A. K. Bousfield, The localization of spectra with respect to homology (correction in Comment. Math. Helv. 58 (1983), 599-600), Topology 18 (1978), 257-281. MR 80m:55006

[4] A. K. Bousfield and D. M. Kan, Homotopy Limits, Completions and Localizations, 2nd corrected edition, Lecture Notes in Mathematics 304, Springer-Verlag, New York, 1987. MR 51:1825

[5] E. S. Devinatz, Morava's change of rings theorem, The Čech Centennial (M. Cenkl and H. Miller, eds.), Contemp. Math. 181, Amer. Math. Soc., Providence, RI, 1995, pp. 93-118. MR 96m:55007

[6] E. S. Devinatz and M. J. Hopkins, Homotopy fixed point spectra for closed subgroups of the Morava stabilizer groups, Topology 43 (2004), 1-47.

[7] J. P. Dixon, M. P. F. du Sautoy, A. Mann, and D. Segal, Analytic Pro- $p$ Groups, 2nd edition, Cambridge Studies in Advanced Mathematics 61, Cambridge University Press, Cambridge 1999. MR 2000m:20039

[8] A. D. Elmendorf, I. Kriz, M. A. Mandell, and J. P. May, Rings, Modules, and Algebras in Stable Homotopy Theory, Amer. Math. Soc. Surveys and Monographs 47, Amer. Math. Soc., Providence, RI, 1997. MR 97h:55006

[9] P. G. Goerss and M. J. Hopkins, Resolutions in model categories, manuscript, 1997.

[10] Simplicial structured ring spectra, manuscript, 1998.

[11] André-Quillen (co-)homology for simplicial algebras over simplicial operads, Une Dégustation Topologique [Topological Morsels]: Homotopy Theory in the Swiss Alps (D. Arlettaz and K. Hess, eds.), Contemp. Math. 265, Amer. Math. Soc., Providence, RI, 2000, pp. 41-85. MR 2001m:18012

[12] - Realizing commutative ring spectra as $E_{\infty}$ ring spectra, manuscript, 2000.

[13] M. J. Hopkins and J. H. Smith, Nilpotence and stable homotopy theory II, Ann. of Math. (2) 148 (1998), 1-49. MR 99h:55009

[14] D. Husemoller and J. C. Moore, Differential graded homological algebra in several variables, Symp. Math. IV, Inst. Naz. di Alta Mat., Academic Press, New York, 1970, pp. 397-429. MR 46:9143

[15] H. R. Miller, On relations between Adams spectral sequences with an application to the stable homotopy of a Moore space, J. of Pure and Applied Alg. 20 (1981), 287-312. MR 82f:55029

[16] C. Rezk, Notes on the Hopkins-Miller theorem, Homotopy Theory via Algebraic Geometry and Group Representations (M. Mahowald and S. Priddy, eds.), Contemp. Math. 220, Amer. Math. Soc., Providence, RI, 1998, pp. 313-366. MR 2000i:55023

[17] J. P. Serre, Galois Cohomology, Springer-Verlag, New York, 1997. MR 98g:12007

[18] S. S. Shatz, Profinite Groups, Arithmetic, and Geometry, Ann. of Math. Stud. 67, Princeton University Press, Princeton, 1972. MR 50:279

[19] P. Symonds and T. Weigel, Cohomology of $p$-adic analytic groups, New Horizons in Pro- $p$ Groups, Progr. Math. 184, Birkhäuser Boston, Boston, 2000, pp. 349-410. MR 2001k:22025

Department of Mathematics, University of Washington, Seattle, Washington 98195

E-mail address: devinatz@math.washington.edu 